\title{
High-temperature X-ray absorption spectroscopy study of thermochromic copper molybdate
}

\author{
Inga Jonane ${ }^{\mathrm{a}, *}$, Andris Anspoks ${ }^{\mathrm{a}}$, Giuliana Aquilanti ${ }^{\mathrm{b}}$, Alexei Kuzmin ${ }^{\mathrm{a}, * *}$ \\ ${ }^{a}$ Institute of Solid State Physics, University of Latvia, Kengaraga street 8, LV-1063 Riga, \\ Latvia \\ ${ }^{b}$ Elettra - Sincrotrone Trieste, ss 14, $\mathrm{km}$ 163.5, 34149 Basovizza, Trieste, Italy
}

\begin{abstract}
X-ray absorption spectroscopy at the $\mathrm{Cu}$ and Mo K-edges was used to study the effect of heating on the local atomic structure and dynamics in copper molybdate $\left(\alpha-\mathrm{CuMoO}_{4}\right)$ in the temperature range from 296 to $973 \mathrm{~K}$. The reverse Monte-Carlo (RMC) method was successfully employed to perform accurate simulations of EXAFS spectra at both absorption edges simultaneously. The method allowed us to determine structural models of $\alpha-\mathrm{CuMoO}_{4}$ being consistent with the experimental EXAFS data. These models were further used to follow temperature dependencies of the local environment of copper and molybdenum atoms and to obtain the mean-square relative displacements for $\mathrm{Cu}-\mathrm{O}$ and $\mathrm{Mo}-\mathrm{O}$ atom pairs. Moreover, the same models were able to interpret strong temperature-dependence of the $\mathrm{Cu} \mathrm{K}$-edge XANES spectra. We found that the local environment of copper atoms is more affected by thermal disorder than that of molybdenum atoms. While the $\mathrm{MoO}_{4}$ tetrahedra behave mostly as the rigid units, a reduction of correlation in atomic motion between copper and axial oxygen atoms occurs upon heating. This dynamic effect seems to be the main responsible for the temperature-induced changes in the $\mathrm{O}^{2-} \rightarrow \mathrm{Cu}^{2+}$ charge transfer processes and, thus, is the origin of the thermochromic properties of $\alpha-\mathrm{CuMoO}_{4}$ upon heating above room temperature.
\end{abstract}

\footnotetext{
*Corresponding author

**Corresponding author. Fax: +371 67132778. Phone: +371) 67251691

Email addresses: inga.jonane@cfi.lu.lv (Inga Jonane), andris.anspoks@cfi.lu.lv (Andris Anspoks), giuliana.aquilanti@elettra.eu (Giuliana Aquilanti), a.kuzmine@cfi.lu.1v (Alexei Kuzmin)
} 
High-temperature X-ray absorption spectroscopy study of thermochromic copper molybdate, Acta Mater. 179 (2019) 26-35.

Keywords: $\mathrm{CuMoO}_{4}$, Thermochromism, XANES, EXAFS, Reverse Monte Carlo simulations 


\section{Introduction}

Transition metal molybdates are well-known materials having a wide range of technological applications [1, 2, 3]. In particular, copper molybdate $\left(\mathrm{CuMoO}_{4}\right)$ has been intensively studied due to its diverse functional properties, including thermochromism, piezochromism, and photoluminescence [4, 5, 6, 7]. It is also known as an efficient oxidation catalyst [8, 9, 10, 11, 12], photocatalyst [13, 14] and n-type photoanode in water splitting photoelectrochemical cells [15]. Furthermore, the properties of the molybdate can be tailored by reducing the particle size or by doping and producing solid solutions [4, 5, 16, 17].

Several structural phases of pure $\mathrm{CuMoO}_{4}$ are known in the literature: $\alpha-\mathrm{CuMoO}_{4}, \beta-\mathrm{CuMoO}_{4}, \gamma-\mathrm{CuMoO}_{4}, \mathrm{CuMoO}_{4}-\mathrm{II}, \mathrm{CuMoO}_{4}-\mathrm{III}, \epsilon-\mathrm{CuMoO}_{4}$ and $\eta$ - $\mathrm{CuMoO}_{4}[18,19,20]$. At ambient pressure, $\mathrm{CuMoO}_{4}$ exists in two phases - $\gamma$-phase with dark brown colour and $\alpha$-phase with green colour. The first order $\alpha$-to- $\gamma$ phase transition occurs upon cooling at $\sim 200 \mathrm{~K}$ and is accompanied by drastic colour change and volume reduction of $12-13 \%$ [18]. The transition to $\gamma$-phase can be also induced by applying pressure [21, 22]. Note that similar colour change from green to brown but of different origin occurs in $\alpha-\mathrm{CuMoO}_{4}$ upon heating up to $\sim 673 \mathrm{~K}[23]$.

Significant changes in the structure and optical properties of $\mathrm{CuMoO}_{4}$ as a result of temperature or pressure treatment make it as a promising material for a wide range of chromic-related applications as, for example, in temperature and/or shock sensors [6] and smart inorganic pigments [24]. $\mathrm{CuMoO}_{4}$ is advantageous at low temperatures due to its reversible thermochromic phase transition [6] and at high temperatures (above $400 \mathrm{~K}$ ) [23], where majority of organic compounds and liquid crystals are unstable.

The green colour of $\mathrm{CuMoO}_{4}$ at room temperature is caused by the transmission window in the range of $\sim 1.9-2.5 \mathrm{eV}$, which separates two optical absorption bands responsible for chromic properties of the material [23]. The red band, located below $\sim 1.9 \mathrm{eV}$, is related to $3 \mathrm{~d}^{9} \rightarrow 4 \mathrm{p}[23]$ or d-d transitions [5, 21, 22] at copper sites, whereas the blue band, located above $\sim 2.5 \mathrm{eV}$, is attributed to the oxygen-to-metal charge transfer [5, 21, 22, 23]. When temperature increases above the room temperature, the transmission window diminishes due to the blue band shift towards longer wavelengths [23].

The structural, optical, electrical and magnetic properties of $\mathrm{CuMoO}_{4}$ were studied previously in $[5,18,21,23,25,26]$. Recently, a negative thermal expansion (NTE) of $\alpha$-CuMoO 4 lattice was proposed at temperatures above 

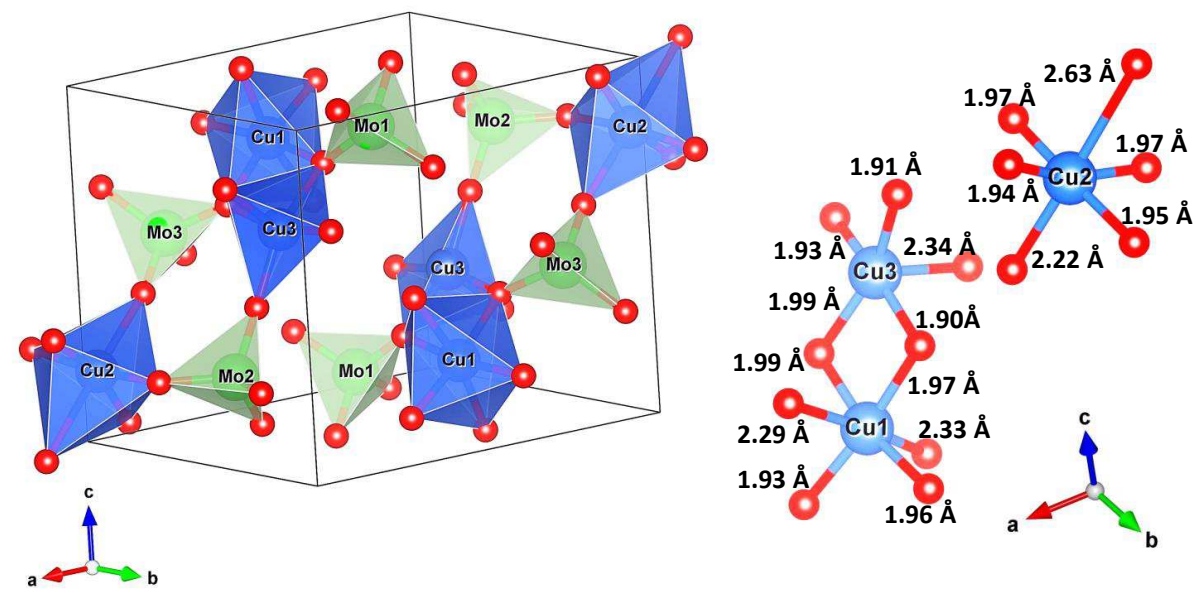

Figure 1: Crystal structure of $\alpha-\mathrm{CuMoO}_{4}$ [18] and three non-equivalent copper atoms with corresponding $\mathrm{Cu}-\mathrm{O}$ distances. Oxygen atoms are red, copper atoms are blue, and molybdenum atoms are green.

room temperature up to $390 \mathrm{~K}$ [27].

In order to understand the relationship between $\alpha-\mathrm{CuMoO}_{4}$ structure and its thermochromic properties, we have performed temperature-dependent Xray absorption spectroscopy (XAS) study at the $\mathrm{Cu}$ and Mo K-edges supported by advanced simulations.

Recently we have used X-ray absorption near-edge structure (XANES) and extended X-ray absorption fine structure (EXAFS) spectroscopy at low temperatures to follow the $\alpha$-to- $\gamma$ and $\gamma$-to- $\alpha$ structural phase transitions in $\mathrm{CuMoO}_{4}$ upon cooling and heating, respectively [28, 29]. In this study, we turn to the behaviour of $\alpha-\mathrm{CuMoO}_{4}$ at high temperatures, that has not been tackled until now using XAS.

Copper molybdate remains in triclinic $P \overline{1}$ symmetry through all temperature range at ambient pressure until the melting point $\sim 1070 \mathrm{~K}[18$ ]. Note that the existence of $\beta-\mathrm{CuMoO}_{4}$ phase with hexagonal symmetry was proposed for temperatures above $840 \mathrm{~K}$ in [30] but its existence was not confirmed in further studies [18]. The structure of $\alpha-\mathrm{CuMoO}_{4}$ is composed of distorted $\mathrm{CuO}_{6}$ octahedra, $\mathrm{CuO}_{5}$ square-pyramids and distorted $\mathrm{MoO}_{4}$ tetrahedra [18]. We will further distinguish three non-equvalent copper (Cu1, $\mathrm{Cu} 2, \mathrm{Cu} 3)$ and molybdenum (Mo1, Mo2, Mo3) atoms with different local environments (Fig. 11).

The interpretation of the X-ray absorption spectrum for such complex 
low-symmetry structure is a challenging task because to account for thermal fluctuations and static disorder the spectrum should be evaluated for all possible geometric configurations and averaged. In this study, we solved this task by advanced simulation-based technique such as the reverse Monte Carlo (RMC) method [31].

First, we applied RMC simulations based on evolutionary algorithm (EA) [31] and ab initio multiple-scattering theory [32] to analyse the temperaturedependent (296-973 K) $\mathrm{Cu}$ and Mo K-edge EXAFS spectra of $\alpha$-CuMoO 4 . As a result, structural models of the material accounting for both static and dynamic (thermal) disorder were obtained. Next, these models were validated using the $\mathrm{Cu}$ K-edge XANES spectra, which show unusually strong temperature dependence, and were employed to determine the radial distribution functions and related structural parameters. Finally, the explanation of the thermochromic behaviour of $\alpha-\mathrm{CuMoO}_{4}$ upon heating above room temperature is proposed.

\section{Experimental details}

High-quality X-ray absorption spectra at the $\mathrm{Cu}(8979 \mathrm{eV})$ and Mo $(20000$ eV) K-edges were recorded in transmission mode at the Elettra XAFS bendingmagnet beamline [33] in the temperature range from $296 \mathrm{~K}$ to $973 \mathrm{~K}$. The storage ring operated in the top-up multibunch mode at the energy $E=$ $2.4 \mathrm{GeV}$ and current $I_{\max }=160 \mathrm{~mA}$. The synchrotron radiation was monochromatized using $\mathrm{Si}(111)$ (for the $\mathrm{Cu} \mathrm{K}$-edge) and $\mathrm{Si}(311)$ (for the Mo K-edge) double-crystal monochromators, and its intensity before and after the sample was measured by two ionization chambers filled with a mixture of $\mathrm{Ar}$, He and $\mathrm{N}_{2}$ gases. Metal foils of copper and molybdenum were used as references for energy calibration.

The samples for the XAS experiments were prepared by mixing $\mathrm{CuMoO}_{4}$ powder, synthesized using solid-state reaction method as described in [28], with boron nitride powder and pressing into pellets. The thickness of the samples for each absorption edge was optimized to get the value of the absorption edge jump $\Delta \mu_{x} \approx 1$. The heating of the samples was performed in vacuum using the l'Aquila-Camerino glass furnace [33]. The sample temperature was stabilized for 15-20 min before each measurement.

Temperature-dependent experimental XANES and EXAFS spectra at the $\mathrm{Cu}$ and Mo K-edges in $\alpha-\mathrm{CuMoO}_{4}$ are shown in Figs. 2 and 3 for selected 

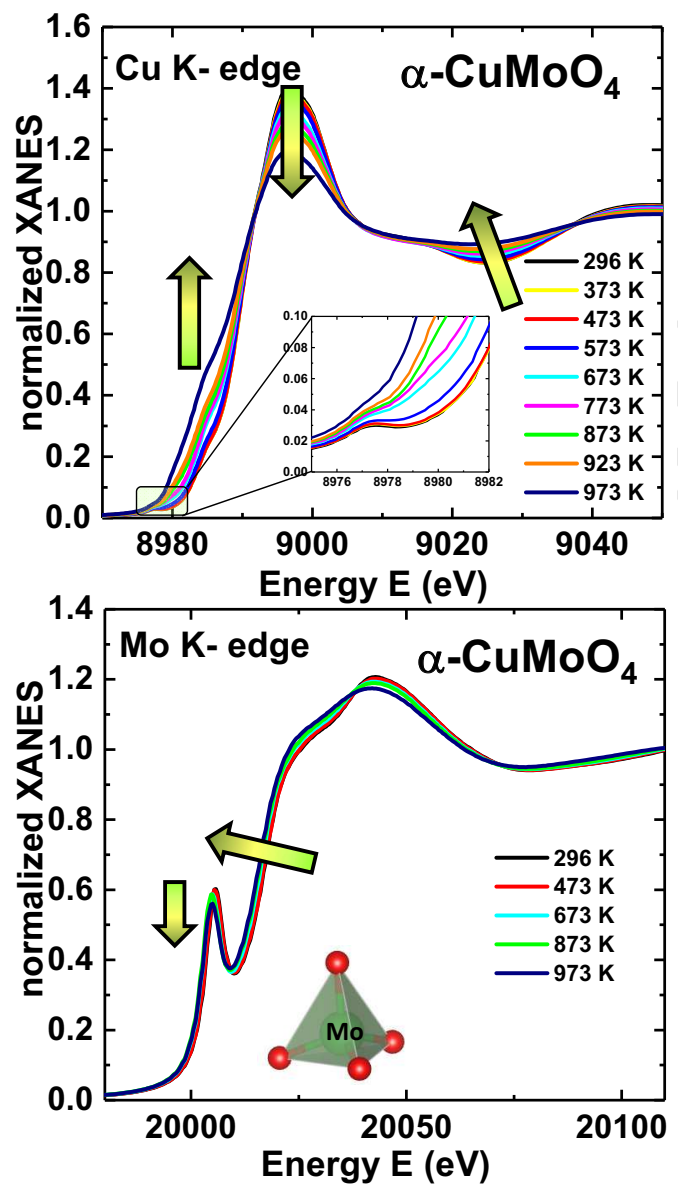

Figure 2: Temperature-dependent experimental $\mathrm{Cu}$ and Mo K-edge XANES of $\alpha-\mathrm{CuMoO}_{4}$.

temperatures. The EXAFS spectra were extracted following the conventional procedure [34].

\section{Data Analysis}

\subsection{Reverse Monte Carlo method}

Temperature-dependent structural information encoded in XAS spectra was extracted from EXAFS data by reverse Monte Carlo calculations coupled with evolutionary algorithm approach (RMC/EA), as implemented in the EvAX code [31]. Our RMC/EA method is based on the iterative random 

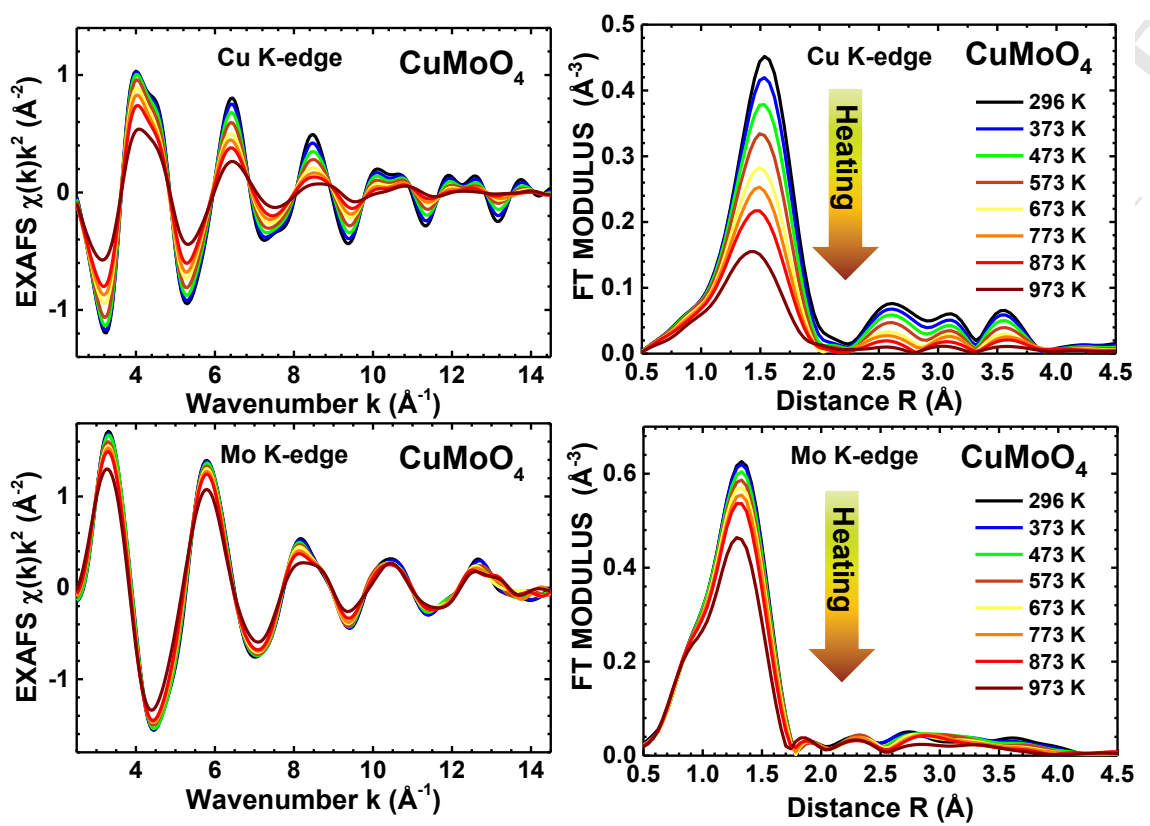

Figure 3: Temperature-dependent experimental $\mathrm{Cu}$ and Mo K-edge EXAFS $\chi(k) k^{2}$ spectra and their Fourier transforms for $\alpha-\mathrm{CuMoO}_{4}$.

changes in the structural model of a material, aimed to minimize the difference between the Morlet wavelet transforms (WTs) [35] of the experimental and calculated configuration-averaged EXAFS spectra. Random atomic displacements introduced by the method allows one to reproduce temperature induced distortions measured in the XAS experiment. Initial structural models for RMC/EA calculations were constructed based on the diffraction data for $\alpha-\mathrm{CuMoO}_{4}$ [18] taking into account temperature-dependent lattice expansion.

The experimental EXAFS spectra at the $\mathrm{Cu}$ and Mo K-edges were analysed simultaneously. The RMC/EA simulations were performed using periodic boundary conditions for a $4 a \times 4 b \times 4 c$ large supercell, containing 2304 atoms. The maximum allowable displacement of atoms at each iteration was set equal to $0.4 \AA$. The configuration-averaged EXAFS spectra were calculated using the ab initio self-consistent real space multiple-scattering (MS) FEFF8.5L code [36]. MS contributions were accounted up to the 5th order. The amplitude reduction factor $S_{0}^{2}$ was equal to one for both absorption edges in all simulations. 


\section{ACCEPTED MANUSCRIPT}
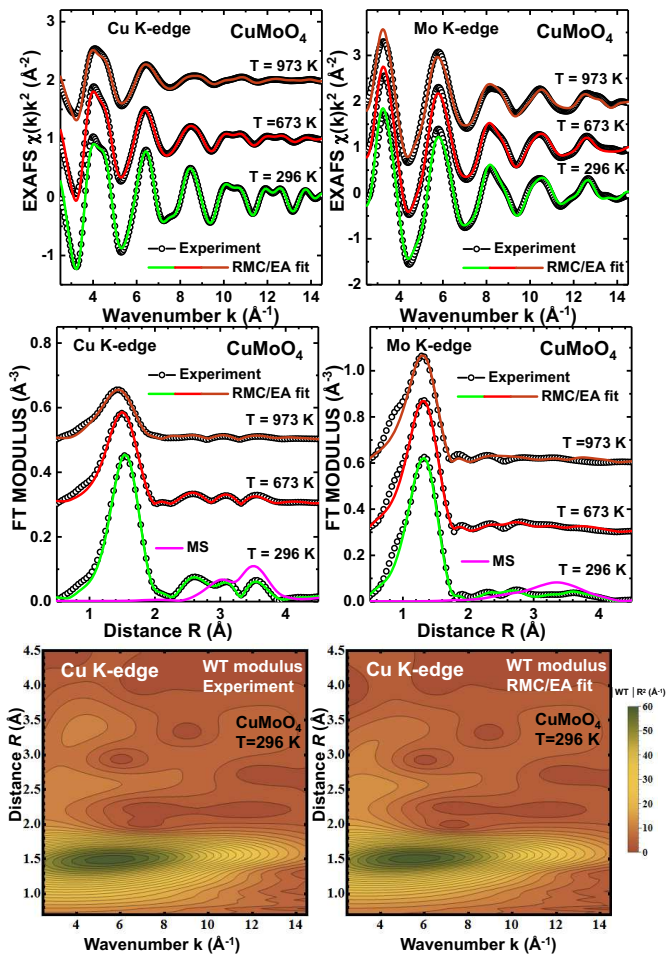

Figure 4: The results of RMC/EA-EXAFS calculations for $\alpha$-CuMoO 4 at selected temperatures: circles - experimental data, lines - calculated spectra. The $\mathrm{Cu}$ and Mo K-edges EXAFS spectra $\chi(k) k^{2}$ are compared in the upper panels and their Fourier transforms (only modulus is shown) - in the middle panels. The Morlet wavelet transforms (WTs) of the experimental and calculated EXAFS spectra at $T=296 \mathrm{~K}$ are also shown in the lower panels.

The comparison between the experimental and calculated EXAFS spectra was performed using the Morlet WT in the $k$-space range from 2.5 to $14.5 \AA^{-1}$ for both edges and in the $R$-space range from 0.7 to $4.5 \AA$ for the $\mathrm{Cu} \mathrm{K}$-edge and from 0.5 to $4.5 \AA$ for the Mo K-edge (Fig. (4). WT allows one to obtain a two-dimensional representation of the EXAFS spectrum in $k$ and $R$ spaces simultaneously that makes the fitting procedure more accurate.

Good agreement between experimental and calculated EXAFS data was obtained after several thousands of iterations. The RMC/EA calculations were repeated several times with different sequences of pseudo-random numbers [31] to improve statistics and to estimate uncertainties of the results. The final structural models were used to determine the radial distribution 
functions (RDFs) $g(R)$ (Fig. 5) for the first coordination shell of copper and molybdenum atoms and to estimate the mean-square relative displacements (MSRDs) $\sigma^{2}$ for the nearest $\mathrm{Cu}-\mathrm{O}$ and $\mathrm{Mo}-\mathrm{O}$ atom pairs within the first shell (Fig. 6).

The MSRDs were calculated from the atomic coordinates using the median absolute deviation (MAD) method [37, 38]. The MAD provides a robust statistic, which is important in the case of RMC when the number of atomic pairs in the simulation box is rather limited. The standard deviation of a distribution was estimated as

$$
\sigma=c \cdot \operatorname{MAD}=c \cdot \operatorname{median}\left(\left|R_{i}-\operatorname{median}(R)\right|\right),
$$

where $c$ is the scalling constant equal to 1.4826 for Gaussian distribution [38].

Temperature dependences of the MSRDs $\sigma^{2}(T)$, including static $\sigma_{s t}^{2}$ and thermal $\sigma_{t h}^{2}$ contributions, were described by the correlated Einstein model [39]

$$
\sigma^{2}(T)=\sigma_{s t}^{2}+\frac{\hbar}{2 \mu \omega_{E}} \operatorname{coth} \frac{\hbar \omega_{E}}{2 k_{B} T},
$$

where $\mu$ is the reduced mass of the atomic pair, $\omega_{E}$ is the Einstein frequency, and $k_{B}$ is the Boltzmann constant. Next, the effective force constants $\kappa$ [40] and the Einstein temperatures $\Theta_{E}$ were evaluated as

$$
\begin{gathered}
\kappa=\mu \omega_{E}^{2}, \\
\Theta_{E}=\frac{\hbar \omega_{E}}{k_{B}} .
\end{gathered}
$$

The obtained values of $\kappa$ and $\Theta_{E}$ for $\mathrm{Cu}-\mathrm{O}$ and $\mathrm{Mo}-\mathrm{O}$ atom pairs are reported in Table 1 .

Table 1: Average values of the Einstein frequency $\omega_{E}$, the Einstein temperature $\Theta_{E}$ and the effective force constant $\kappa$ calculated from the temperature dependences of the MSRD factors $\sigma^{2}(T)$ shown in Fig. 6 for the four nearest $\mathrm{Cu}-\mathrm{O}$ and $\mathrm{Mo}-\mathrm{O}$ atom pairs in $\alpha$ $\mathrm{CuMoO}_{4}$.

\begin{tabular}{lccc}
\hline Atom pair & $\begin{array}{c}\text { Einstein frequency } \\
\omega_{E}[\mathrm{THz}]\end{array}$ & $\begin{array}{c}\text { Einstein temperature } \\
\Theta_{E}[\mathrm{~K}]\end{array}$ & $\begin{array}{c}\text { Effective force constant } \\
\kappa[\mathrm{N} / \mathrm{m}]\end{array}$ \\
\hline $\mathrm{Cu}-\mathrm{O}$ & $55 \pm 6$ & $422 \pm 42$ & $65 \pm 13$ \\
$\mathrm{Mo}-\mathrm{O}$ & $122 \pm 20$ & $932 \pm 153$ & $341 \pm 112$ \\
\hline
\end{tabular}




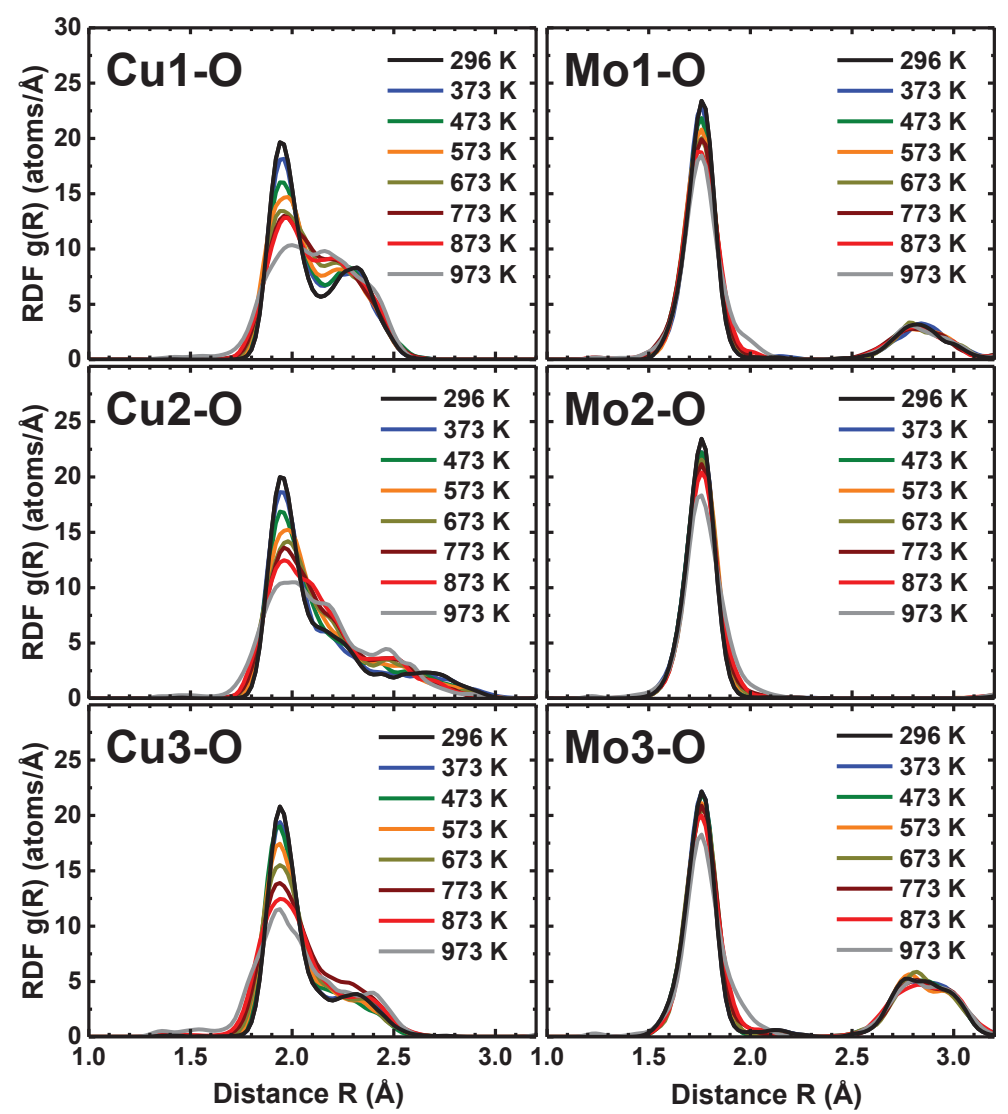

Figure 5: Radial distribution functions for three non-equivalent $\mathrm{Cu}-\mathrm{O}$ and $\mathrm{Mo}-\mathrm{O}$ atom pairs in $\alpha-\mathrm{CuMoO}_{4}$ reconstructed by the RMC/EA method.

\subsection{XANES calculations}

The most pronounced thermal effect in XANES of $\alpha-\mathrm{CuMoO}_{4}$ was observed at the $\mathrm{Cu}$ K-edge (Fig. 2). To interpret this phenomenon, XANES calculations were performed by the ab initio full-multiple-scattering FDMNES code [41, 42] employing muffin-tin (10\% overlap) self-consistent potential. The dipole and quadrupole transitions were taken into account. The cluster with the radius of $4.0 \AA$ was constructed around each absorbing copper atom to account for all atoms producing significant contributions into the FTs in Fig. 3, All calculated XANES spectra were broadened by the $\mathrm{Cu}$ K-edge core level width $\Gamma_{\text {core }}=1.55 \mathrm{eV}$.

The calculated $\mathrm{Cu}$ K-edge XANES spectra for three non-equivalent cop- 


\section{ACCEPTED MANUSCRIPT}

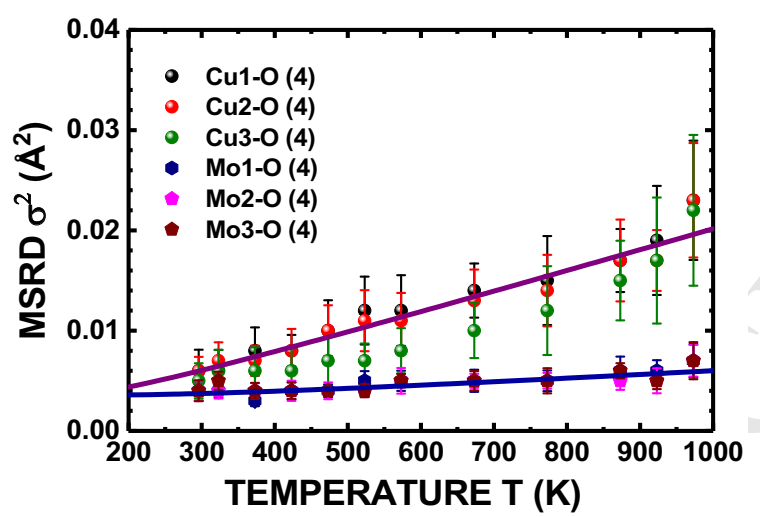

Figure 6: Temperature dependence of the MSRD factors $\sigma^{2}$ for the four shortest metaloxygen $(\mathrm{Cu}-\mathrm{O}$ and $\mathrm{Mo}-\mathrm{O})$ bonds in $\alpha-\mathrm{CuMoO}_{4}$. Best fitted correlated Einstein models are shown by solid lines.

per atoms (Cu1, Cu2, Cu3) in the average crystallographic structure at $296 \mathrm{~K}$, known from the diffraction data [18], are shown in Fig. 7(a). The XANES spectrum for $\mathrm{Cu} 2 *$, which corresponds to the $\mathrm{Cu} 2$ atom without two axial oxygen atoms, was also evaluated to test the influence of oxygen vacancies on the spectrum shape.

Next, we performed XANES calculations for the structural models obtained by the RMC/EA-EXAFS simulations, which include thermal disorder effects. In this case, the $\mathrm{Cu}$ K-edge XANES spectra were calculated for 48 copper atoms located in the central $2 a \times 2 b \times 2 c$ part of the supercell (Fig. 8), and the averaged XANES spectra calculated from three independent RMC/EA simulations are reported for four selected temperatures (296, 673, 873 and $973 \mathrm{~K}$ ) in Fig. 7(c). Note that some atomic configurations result in the XANES spectra deviating rather strongly from the average one; however, their percentage is small.

Finally, the $\mathrm{Cu}$ K-edge XANES spectra were calculated for three selected temperatures using the experimental values of the temperature-dependent lattice parameters from the diffraction data [18] and are shown in Fig. [(d).

\section{Results and discussion}

\subsection{EXAFS data analysis}

Temperature-dependent experimental EXAFS spectra $\chi(k) k^{2}$ at the $\mathrm{Cu}$ and Mo K-edges in $\alpha-\mathrm{CuMoO}_{4}$ are shown in Fig. 3 at selected temperatures. 


\section{ACCEPTED MANUSCRIPT}

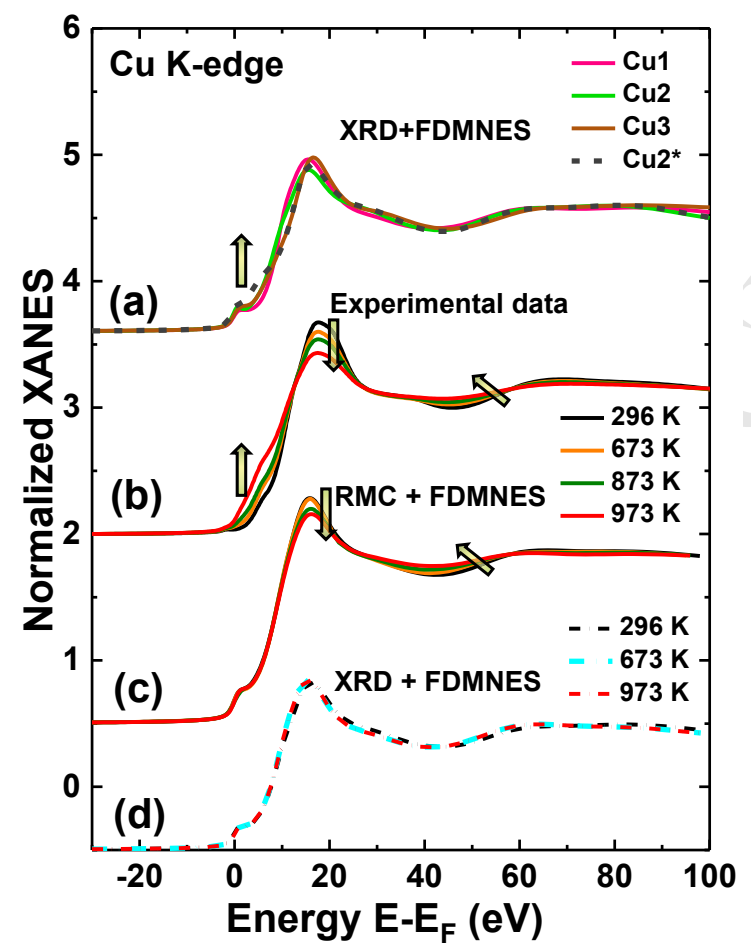

Figure 7: The $\mathrm{Cu}$ K-edge XANES spectra of $\alpha-\mathrm{CuMoO}_{4}$. (a) The XANES spectra calculated for non-equvalent $\mathrm{Cu} 1, \mathrm{Cu} 2$ and $\mathrm{Cu} 3$ atoms in the average structure at $296 \mathrm{~K}$ known from diffraction measurements [18]. The XANES spectrum for $\mathrm{Cu} 2 *$ corresponds to the case of the $\mathrm{Cu} 2$ atom without two axial oxygen atoms. (b) The experimental XANES at four selected temperatures. (c) The averaged XANES spectra calculated for the RMC/EA structure models at four selected temperatures. (d) The averaged XANES spectra calculated for the XRD structure models at three selected temperatures taking into account molybdate lattice expansion [18].

Both sets of spectra are dominated by the first coordination shell contributions, while the structural peaks in the Fourier transforms (FTs) are well observable up to $\sim 4 \AA$. One can also see that thermal disorder produces a stronger effect on the $\mathrm{Cu}$ K-edge data.

The results of RMC/EA simulations for selected temperatures are shown in Fig. 4. Good agreement between experimental and calculated EXAFS spectra was obtained for both edges simultaneously at all temperatures supporting the reliability of extracted structural data.

The first peak in FT of the $\mathrm{Cu}$ K-edge EXAFS spectrum at $\sim 1.5 \AA$ and of the Mo K-edge EXAFS spectrum at $\sim 1.3 \AA$ corresponds to the 

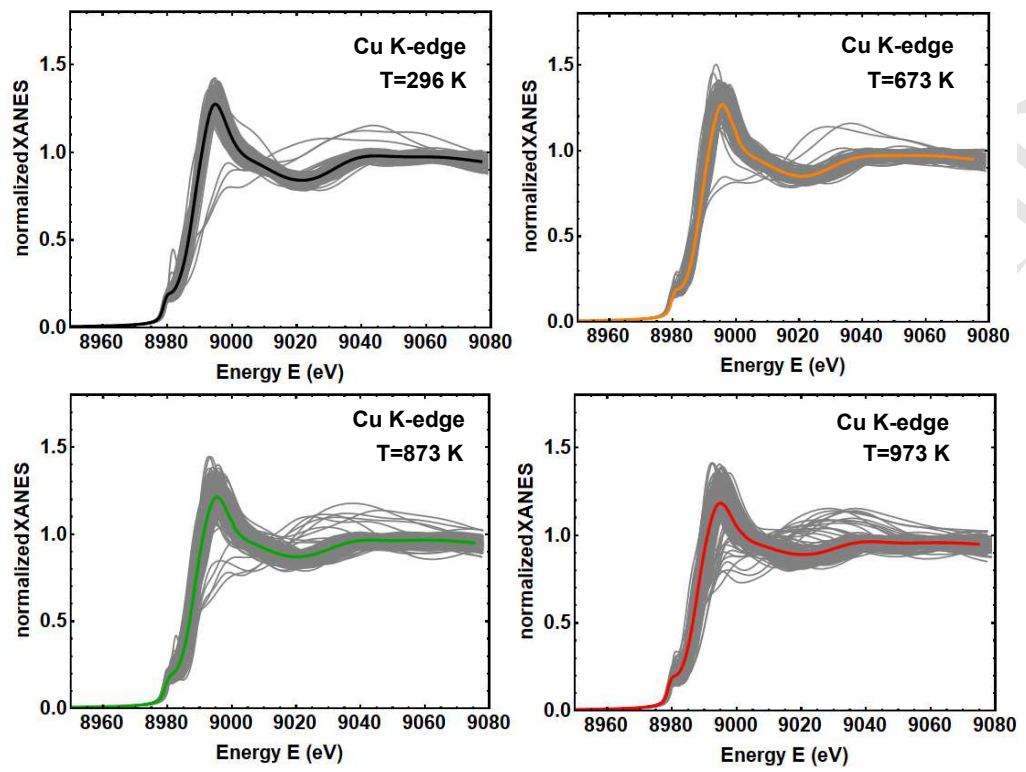

Figure 8: The calculated $\mathrm{Cu}$ K-edge XANES spectra for structural models obtained in the RMC/EA-EXAFS simulations for four selected temperatures. Gray lines correspond to XANES spectra of individual copper atoms in the supercell, coloured lines show the configuration-averaged XANES spectra.

single-scattering contribution from nearest oxygen atoms forming the first coordination shell around $\mathrm{Cu}$ and Mo ions. Next peaks are due to a superposition of contributions from more distant atoms and MS effects. The total MS signals were calculated at both edges, and their FTs are shown in Fig. 4 at $T=296 \mathrm{~K}$. Their contributions are important above $\sim 2.2 \AA$ and should be taken into account to reproduce accurately the experimental signals. Note also that the presence of MS contributions introduces angular sensitivity into EXAFS and thus is essential for atomic structure reconstruction in RMC/EA simulations.

The final sets of atomic coordinates obtained in RMC/EA simulations were used to calculate partial radial distribution functions $g_{\mathrm{Cu}-\mathrm{O}}(R)$ and $g_{\mathrm{Mo}-\mathrm{O}}(R)$ for all non-equivalent metal atoms (Fig. 5 ). Note that the ability to distinguish the local environment around any atom of interest is a big advantage of the RMC/EA method compared to conventional fitting methods.

An increase in temperature has a strong effect on the $\mathrm{Cu}-\mathrm{O}$ distributions, leading to the broadening of the peaks due to an increase in the amplitudes of thermal vibrations of the atoms. The first peak in the $\mathrm{Cu}-\mathrm{O}$ distributions 
located at $\sim 1.95 \AA$ corresponds to the nearest 4 oxygen atoms and is close for three non-equivalent copper atoms. The second peak at $\sim 2.3-2.7 \AA$ is due to more distant axially placed oxygen atoms of the first coordination shell (two oxygens in $\mathrm{Cu} 1$ and $\mathrm{Cu} 2$ octahedra or one oxygen in $\mathrm{Cu} 3$ square-pyramids). The $\mathrm{Cu} 2$ atoms have the most distorted local environment because of the sixth weakly bound oxygen atom located at $\sim 2.63 \AA$ (Fig. 1).

The local environment around molybdenum atoms is less affected by an increase of temperature due to relatively strong bonding between molybdenum and oxygen atoms within $\mathrm{MoO}_{4}$ tetrahedra. The $\mathrm{Mo}-\mathrm{O}$ distance is equal to $\sim 1.78 \AA$ for all three non-equivalent molybdenum atoms, while some asymmetry of the Mo-O peak shape becomes visible above $\sim 800 \mathrm{~K}$ (Fig. 5). Note that there are groups of one and two oxygen atoms around Mo1 and Mo3 atoms, respectively, located at $\sim 2.8 \AA$. However, being rather closely located, these oxygen atoms are not bound directly to the molybdenum atoms.

Temperature dependence of the MSRD factors $\sigma^{2}$ for the groups of four shortest $\mathrm{Cu}-\mathrm{O}$ and $\mathrm{Mo}-\mathrm{O}$ bonds is shown in Fig. 6 together with the correlated Einstein models obtained using Eq. 2. The MSRDs of the $\mathrm{Cu}_{-}^{-}$ $\mathrm{O}$ atom pairs are rather close for three non-equivalent copper atoms and are larger than those for stronger $\mathrm{Mo}-\mathrm{O}$ bonds. Respectively, the parameter of the Einstein model in Table 1 is significantly smaller for the $\mathrm{Cu}-\mathrm{O}$ bonds than for the $\mathrm{Mo}-\mathrm{O}$ bonds. In the temperature range of 296-973 K, the $\mathrm{Cu}-\mathrm{O}$ MSRD factor for the four shortest $\mathrm{Cu}-\mathrm{O}$ bonds increases from $0.006 \pm 0.002 \AA^{2}$ to $0.022 \pm 0.006 \AA^{2}$, whereas the Mo-O MSRD factor increases from $0.004 \pm 0.001 \AA^{2}$ to only $0.007 \pm 0.002 \AA^{2}$. This means that the $\mathrm{MoO}_{4}$ tetrahedra can be considered as rigid units.

The MSRD factors for longer $\mathrm{Cu}-\mathrm{O}$ bonds, linking copper atoms with axially located oxygen atoms, are generally larger than those for the short $\mathrm{Cu}-\mathrm{O}$ bonds. However, our EXAFS analysis was not able to discriminate them with enough accuracy due to the limited sensitivity of EXAFS to distant oxygen atoms, whose contribution to the total EXAFS spectrum is weak.

When RMC simulations are used to fit EXAFS spectra, one can validate the obtained sets of atomic coordinates also against diffraction data. To do this, we projected coordinates of all atoms in the supercell into a single unit cell and calculated the average positions for each non-equivalent atom. Thus obtained average positions of atoms correspond to the Wyckoff positions, so that the diffraction pattern can be calculated and compared with the experimental one known from the diffraction experiment [18]. An example 
of such procedure is shown in Fig. 9 for $\alpha$-CuMoO 4 at $973 \mathrm{~K}$. As one can see, our RMC/EA simulations reproduce rather well the average positions of atoms and, as a result, the diffraction pattern.

\subsection{XANES analysis}

The $\mathrm{Cu}$ and Mo K-edge XANES spectra in $\alpha-\mathrm{CuMoO}_{4}$ are shown in Fig. 2. Their origin is due to dipole allowed $1 \mathrm{~s}(\mathrm{Cu} / \mathrm{Mo}) \rightarrow \mathrm{np}$ transitions in the presence of the core hole at the $1 \mathrm{~s}(\mathrm{Cu} / \mathrm{Mo})$ core level. Besides, there is a strong pre-edge peak at the Mo K-edge located at $\sim 20006 \mathrm{eV}$. It is due to the $1 \mathrm{~s}(\mathrm{Mo}) \rightarrow 4 \mathrm{~d}(\mathrm{Mo})+2 \mathrm{p}(\mathrm{O})$ transition, and its intensity depends on the $4 \mathrm{~d}(\mathrm{Mo}) / 2 \mathrm{p}(\mathrm{O})$ orbital mixing and the number of unoccupied states $\left(\mathrm{Mo}^{6+}\left(4 \mathrm{~d}^{0}\right)\right)$ [43, 44]. On the contrary, the pre-edge peak at the $\mathrm{Cu}$ K-edge is very weak and is located at $\sim 8978 \mathrm{eV}$. It is due to the $1 \mathrm{~s}(\mathrm{Cu}) \rightarrow 3 \mathrm{~d}(\mathrm{Cu})+2 \mathrm{p}(\mathrm{O})$ transition [45, 46], which is weaker in six-fold or fivefold coordination than in the tetrahedral one, but the small intensity of the peak is mainly due to the limited number of unoccupied states $\left(\mathrm{Cu}^{2+}\left(3 \mathrm{~d}^{9}\right)\right)$. Note that the pre-edge peak at the $\mathrm{Cu}$ K-edge becomes even less resolved at high temperatures (see the inset in Fig. 2(top panel)).

Recently, we have shown that there is a correlation between the preedge peak intensity at the Mo K-edge and a degree of the distortion of molybdenum-oxygen coordination polyhedra in $\mathrm{CuMoO}_{4}$ [28]. Weak temperature dependence of the experimental Mo K-edge XANES in Fig. 2(bottom panel) indicates that molybdenum atoms remain in tetrahedral coordination at all studied temperatures, in agreement with the diffraction data [18]. The only experimentally observed effect is related to the thermal expansion of the lattice, which leads to a decrease of the oscillation frequency above the edge.

At the same time, the $\mathrm{Cu}$ K-edge XANES spectra show a rather strong temperature dependence (Fig. 2(top panel)). In particular, upon the temperature increase from 296 to $973 \mathrm{~K}$, the shoulder appears at $\sim 8985 \mathrm{eV}$, the main peak at $\sim 8997 \mathrm{eV}$ becomes smaller, and some increase of the absorption occurs at $\sim 9025 \mathrm{eV}$. We have found that such strong variations of the $\mathrm{Cu}$ K-edge XANES cannot be related simply to the lattice expansion. The XANES calculations performed for the equilibrium static structure [18] taking into account the thermal expansion result in close XANES spectra (Fig. $7(\mathrm{~d})$ ), which differ only in the oscillation frequency above the edge due to the expected increase of interatomic distances. It is important to note that the model based on the equilibrium static structure is not able to reproduce temperature dependence observed in the experiment and, in fact, deviates 
significantly from the experimental XANES spectra at high-temperatures. This result suggests that thermal disorder should be explicitly included in the $\mathrm{Cu}$ K-edge XANES model.

There are a few studies known in the literature that demonstrated a sensitivity of XANES spectra to thermal disorder [47, 48, 49, 50, 51]. Here, we used the results of RMC/EA-EXAFS simulations to interpret XANES temperature dependence. This approach has an advantage since atomic configurations employed in the XANES calculations are first validated against EXAFS spectra and intrinsically include correlation effects in atomic motion.

The simulated $\mathrm{Cu}$ K-edge XANES spectra corresponding to RMC/EA structural models are shown in Fig. 8. While some configurations produce quite different XANES, the configuration-averaged XANES spectra are close to the experimental data. They reproduce well temperature dependence observed experimentally, including the behaviour of the main peak at $\sim$ $8997 \mathrm{eV}$ and the fine structure at $\sim 9025 \mathrm{eV}$ (compare Fig. 7(b) and (c)). However, the origin of the shoulder increase at $\sim 8985 \mathrm{eV}$ remains puzzling.

Theoretical calculations suggest that the shoulder intensity increases when the octahedral coordination of copper atoms transforms into square-like by loosing two axial oxygen (O5, O6) atoms (see Cu2* model in Fig. 7(a)). Since these oxygen atoms are always present in the structure, we propose that this effect is of dynamic origin. Two axial oxygen atoms are weakly bound to copper atoms but, at the same time, are strongly bound to molybdenum atoms, participating in the formation of rigid $\mathrm{MoO}_{4}$ tetrahedra. We expect that an increase of the librational motion of $\mathrm{MoO}_{4}$ tetrahedra at high temperatures weakens interaction between axial oxygen atoms and copper atoms, making their relative motion uncorrelated and, thus, reducing their contribution to the $\mathrm{Cu}$ K-edge XANES. Unfortunately, our RMC/EA simulations are not sensitive enough to the contribution of axial oxygen atoms, which is rather weak. Therefore, the obtained atomic configurations are not able to reproduce accurately the temperature dependence of the the $\mathrm{Cu}$ K-edge shoulder at $\sim 8985 \mathrm{eV}$.

\subsection{Thermochromic properties}

It is known that thermochromic behaviour of $\alpha-\mathrm{CuMoO}_{4}$ above room temperature is governed by an interplay between two absorption bands due to the band gap in the blue and due to $\mathrm{Cu}^{2+}\left(\mathrm{d}^{9}\right) \mathrm{d}-\mathrm{d}$ transition in the red [5, 21, 23, 22]. While there is a consensus on the origin of the red band located below $\sim 1.9 \mathrm{eV}[5,21,22]$, the origin of the band gap $(\sim 2.5 \mathrm{eV})$ is 
still not fully understood and was attributed to $\mathrm{O}^{2-} \rightarrow \mathrm{Mo}^{6+}[5]$ or $\mathrm{O}^{2-} \rightarrow \mathrm{Cu}^{2+}$ [21, 22] charge transfer processes. Note that the colour change from green to brown upon heating is caused by the red shift of the band gap.

The band gaps of several molybdates with tetrahedral coordination of molybdenum atoms were estimated from reflectivity spectra and are equal to $4.3 \mathrm{eV}$ for $\alpha-\mathrm{ZnMoO}_{4}$ [52], $5.1 \mathrm{eV}$ for $\mathrm{CaMoO}_{4}$ and $5.3 \mathrm{eV}$ for $\mathrm{SrMoO}_{4}$ [53]. In these materials the band gap is determined by electronic transitions in $\mathrm{MoO}_{4}^{2-}$ oxyanionic groups from the top of the valence band formed mainly by $\mathrm{O} 2 \mathrm{p}$ states to the conduction band dominated by Mo $4 \mathrm{~d}$ states [54]. For comparison, an optical band gap of $3.17 \mathrm{eV}$ was found for $\beta-\mathrm{ZnMoO}_{4}$ with distorted octahedral coordination of molybdenum ions [55]. Therefore, it seems reasonable that the blue band in $\alpha-\mathrm{CuMoO}_{4}$ located at even lower energy of about $2.5 \mathrm{eV}$ is not related to $\mathrm{O}^{2-} \rightarrow \mathrm{Mo}^{6+}$ charge transfer transition.

As we have shown (Figs. 5 and 6), $\mathrm{MoO}_{4}$ tetrahedra in $\alpha-\mathrm{CuMoO}_{4}$ behave as rigid units with strong $\mathrm{Mo}-\mathrm{O}$ bonding and weak temperature dependence compared to $\mathrm{CuO}_{6}$ octahedra, where the $\mathrm{Cu}-\mathrm{O}$ axial bonds undergo the largest changes. Since axial oxygen atoms around copper simultaneously belong to $\mathrm{MoO}_{4}$ tetrahedra, the librational movement of rigid $\mathrm{MoO}_{4}$ units weakens the interaction between copper and axial oxygen atoms at high temperatures, which is observed as an increase of the shoulder at $8985 \mathrm{eV}$ at the Cu K-edge XANES (Fig. 2(top panel)). Therefore, we propose that this dynamic effect, complemented by a small lattice expansion, is responsible for an increase of the band gap and for the colour change of $\alpha-\mathrm{CuMoO}_{4}$ from green to brown upon heating.

\section{Conclusions}

The local atomic structure and dynamics were studied in thermochromic $\alpha$-CuMoO 4 using $\mathrm{X}$-ray absorption spectroscopy in the temperature range from 296 to $973 \mathrm{~K}$.

The RMC/EA-EXAFS method was successfully employed to perform accurate and simultaneous analysis of the $\mathrm{Cu}$ and Mo K-edge EXAFS spectra of polycrystalline $\alpha-\mathrm{CuMoO}_{4}$. The partial radial distribution functions $g_{\mathrm{Cu}-\mathrm{O}}(R)$ and $g_{\mathrm{Mo}-\mathrm{O}}(R)$ were obtained for all non-equivalent atoms in the unit cell, providing information on the temperature dependence of metaloxygen coordination polyhedra distortion. Our RMC/EA-EXAFS simulations were also able to reproduce rather well the average positions of atoms (the Wyckoff positions) in agreement with known diffraction data [18]. 
The mean-square relative displacements $\sigma^{2}$ for $\mathrm{Cu}-\mathrm{O}$ and $\mathrm{Mo}-\mathrm{O}$ atom pairs were determined within the first coordination shell of metal atoms. The values of the Einstein frequency $\omega_{E}$, the Einstein temperature $\Theta_{E}$ and the effective force constant $\kappa$ were calculated from the temperature dependences of $\sigma^{2}(T)$ for the four nearest $\mathrm{Cu}-\mathrm{O}$ and $\mathrm{Mo}-\mathrm{O}$ atom pairs. The obtained results support the rigidity of $\mathrm{MoO}_{4}$ tetrahedra.

We found that both the $\mathrm{Cu}$ and Mo K-edge XANES spectra are sensitive to thermal disorder. However, the Mo K-edge XANES shows weak temperature dependence caused by the lattice thermal expansion, whereas thermal disorder affects strongly the $\mathrm{Cu}$ K-edge XANES. To address this problem, structural models obtained by RMC/EA simulations were used to calculate the configuration-averaged $\mathrm{Cu}$ K-edge XANES using the ab initio full-multiple-scattering theory. Thus calculated XANES spectra are in agreement with the experimental ones and reproduce their temperature-dependent behaviour.

At the same time, we found that the interpretation of the temperature dependence of the $\mathrm{Cu}$ K-edge shoulder at $\sim 8985 \mathrm{eV}$ requires a more sophisticated model, accounting for the reduction of correlation in atomic motion between copper and axial oxygen atoms. These results suggest that the thermochromic properties of $\alpha-\mathrm{CuMoO}_{4}$ upon heating above room temperature can be attributes to a change in the $\mathrm{O}^{2-} \rightarrow \mathrm{Cu}^{2+}$ charge transfer processes.

\section{Acknowledgements}

Financial support provided by Scientific Research Project for Students and Young Researchers Nr. SJZ/2017/5 and SJZ/2018/1 realized at the Institute of Solid State Physics, University of Latvia is greatly acknowledged. The work was also supported by philanthropist MikroTik and administrated by the University of Latvia Foundation. The experiment at the Elettra synchrotron was performed within the project No. 20150303.

[1] A. M. Kaczmarek, R. Van Deun, Rare earth tungstate and molybdate compounds - from 0D to 3D architectures, Chem. Soc. Rev. 42 (2013) 8835-8848. doi:10.1039/C3CS60166H.

[2] L. Zhang, S. Zheng, L. Wang, H. Tang, H. Xue, G. Wang, H. Pang, Fabrication of metal molybdate micro/nanomaterials for electrochemical energy storage, Small 13 (2017) 1700917. doi:10.1002/smll.201700917. 
[3] Q. Li, Y. Xu, S. Zheng, X. Guo, H. Xue, H. Pang, Recent progress in some amorphous materials for supercapacitors, Small 14 (2018) 1800426. doi:10.1002/smll.201800426.

[4] L. Robertson, N. Penin, V. Blanco-Gutierrez, D. Sheptyakov, A. Demourgues, $\mathrm{M}$. Gaudon, $\mathrm{CuMo}_{0.9} \mathrm{~W}_{0.1} \mathrm{O}_{4}$ phase transition with thermochromic, piezochromic, and thermosalient effects, J. Mater. Chem. C 3 (2015) 2918-2924. doi:10.1039/C4TC02463J.

[5] M. Gaudon, C. Carbonera, A. E. Thiry, A. Demourgues, P. Deniard, C. Payen, J. F. Létard, S. Jobic, Adaptable thermochromism in the $\mathrm{CuMo}_{1-x} \mathrm{~W}_{x} \mathrm{O}_{4}$ series $(0 \leq x<0.1)$ : a behavior related to a first-order phase transition with a transition temperature depending on $\mathrm{x}$, Inorg. Chem. 46 (2007) 10200-10207. doi:10.1021/ic701263c.

[6] V. Blanco-Gutierrez, L. Cornu, A. Demourgues, M. Gaudon, $\mathrm{CoMoO}_{4} / \mathrm{CuMo}_{0.9} \mathrm{~W}_{0.1} \mathrm{O}_{4}$ mixture as an efficient piezochromic sensor to detect temperature/pressure shock parameters, ACS Appl. Mater. Interfaces 7 (2015) 7112-7117. doi:10.1021/am508652h.

[7] W. Zhang, J. Yin, F. Min, L. Jia, D. Zhang, Q. Zhang, J. Xie, Preparation and photoluminescence properties of $\mathrm{MMoO}_{4}(\mathrm{M}=\mathrm{Cu}, \mathrm{Ni}, \mathrm{Zn})$ nano-particles synthesized via electrolysis, J. Mol. Struct. 1127 (2017) 777-783. doi:10.1016/j.molstruc.2016.08.020.

[8] K. Makarevich, N. Lebukhova, P. Chigrin, N. Karpovich, Catalytic properties of $\mathrm{CuMoO}_{4}$ doped with $\mathrm{Co}, \mathrm{Ni}$, and $\mathrm{Ag}$, Inorg. Mater. 46 (2010) 1359-1364. doi:10.1134/S0020168510120162.

[9] P. Chigrin, N. Lebukhova, A. Y. Ustinov, Structural transformations of $\mathrm{CuMoO}_{4}$ in the catalytic oxidation of carbon, Kinet. Catal. 54 (2013) 76-80. doi:10.1134/S0023158413010035.

[10] P. G. Chigrin, N. V. Lebukhova, A. Y. Ustinov, Kinetics of soot oxidation catalyzed by $\mathrm{CuMoO}_{4}$, React. Kinet. Mech. Cat. 113 (2014) 1-17. doi:10.1007/s11144-014-0754-7.

[11] B. Wang, K. Zhou, S. Jiang, Y. Hu, Z. Gui, The application of transition metal molybdates $\left(\mathrm{AMoO}_{4}, \mathrm{~A}=\mathrm{Co}, \mathrm{Ni}, \mathrm{Cu}\right)$ as additives in acrylonitrile-butadiene-styrene with improved flame retardant and 
smoke suppression properties, Polym. Adv. Technol. 25 (2014) 14191425. doi:10.1002/pat.3380.

[12] E. Soltys, K. K. Urazov, T. Kharlamova, O. Vodyankina, Redox and catalytic properties of copper molybdates with various composition, Kinet. Catal. 59 (2018) 58-69. doi:10.1134/S0023158418010111.

[13] M. D. Ward, J. F. Brazdil, S. Mehandru, A. B. Anderson, Methane photoactivation on copper molybdate: an experimental and theoretical study, J. Phys. Chem. 91 (1987) 6515-6521. doi:10.1021/j100310a019.

[14] T. Ghorai, D. Dhak, S. Dalai, P. Pramanik, Effect of photocatalytic activities of nano-sized copper molybdate $\left(\mathrm{CuMoO}_{4}\right)$-doped bismuth titanate $\left(\mathrm{Bi}_{2} \mathrm{Ti}_{4} \mathrm{O}_{11}\right)$ (CMBT) alloy, Mater. Res. Bull. 43 (2008) 17701780. doi:10.1016/j.materresbull.2007.07.009.

[15] J. C. Hill, Y. Ping, G. A. Galli, K.-S. Choi, Synthesis, photoelectrochemical properties, and first principles study of n-type $\mathrm{CuW}_{1-x} \mathrm{Mo}_{x} \mathrm{O}_{4}$ electrodes showing enhanced visible light absorption, Energy Environ. Sci. 6 (2013) 2440-2446. doi:10.1039/C3EE40827B.

[16] M. Benchikhi, R. El Ouatib, S. Guillemet-Fritsch, L. Er-Rakho, B. Durand, Characterization and photoluminescence properties of ultrafine copper molybdate $\left(\alpha-\mathrm{CuMoO}_{4}\right)$ powders prepared via a combustion-like process, Int. J. Min. Met. Mater. 23 (2016) 1340-1345. doi:10.1007/s12613-016-1356-3.

[17] M. Benchikhi, R. El Ouatib, S. Guillemet-Fritsch, L. Er-Rakho, B. Durand, Investigation of structural transition in molybdates $\mathrm{CuMo}_{1-x} \mathrm{~W}_{x} \mathrm{O}_{4}$ prepared by polymeric precursor method, Process. Appl. Ceram. 11 (2017) 21-26. doi:10.2298/PAC1701021B.

[18] M. Wiesmann, H. Ehrenberg, G. Miehe, T. Peun, H. Weitzel, H. Fuess, p-T phase diagram of $\mathrm{CuMoO}_{4}$, J. Solid State Chem. 132 (1997) 88-97. doi:10.1006/jssc.1997.7413.

[19] J. Baek, A. S. Sefat, D. Mandrus, P. S. Halasyamani, A new magnetically ordered polymorph of $\mathrm{CuMoO}_{4}$ : synthesis and characterization of $\varepsilon-\mathrm{CuMoO}_{4}$, Chem. Mater. 20 (2008) 3785-3787. doi:10.1021/cm801162j. 
[20] S. Hara, H. Sato, Structure and magnetism of novel copper molybdenum oxides $\eta-\mathrm{CuMoO}_{4}$ and $\beta-\mathrm{Cu}_{3} \mathrm{Mo}_{2} \mathrm{O}_{9}$, J. Phys. Soc. Japan 82 (2013) 054802. doi:10.7566/JPSJ.82.054802.

[21] F. Rodríguez, D. Hernández, J. Garcia-Jaca, H. Ehrenberg, $\mathrm{H}$. Weitzel, Optical study of the piezochromic transition in $\mathrm{CuMoO}_{4}$ by pressure spectroscopy, Phys. Rev. B 61 (2000) 16497-16501. doi:10.1103/PhysRevB.61.16497.

[22] D. Hernández, F. Rodriguez, J. Garcia-Jaca, H. Ehrenberg, H. Weitzel, Pressure-dependence on the absorption spectrum of $\mathrm{CuMoO}_{4}$ : study of the green $\rightarrow$ brownish-red piezochromic phase transition at $2.5 \mathrm{kbar}$, Physica B 265 (1999) 181-185. doi:10.1016/S0921-4526(98)01366-0.

[23] G. Steiner, R. Salzer, W. Reichelt, Temperature dependence of the optical properties of $\mathrm{CuMoO}_{4}$, Fresenius J. Anal. Chem. 370 (2001) 731-734. doi:10.1007/s002160000630,

[24] I. Yanase, T. Mizuno, H. Kobayashi, Structural phase transition and thermochromic behavior of synthesized W-substituted $\mathrm{CuMoO}_{4}$, Ceram. Int. 39 (2013) 2059-2064. doi:10.1016/j.ceramint.2012.08.059.

[25] H. Ehrenberg, H. Weitzel, H. Paulus, M. Wiesmann, G. Wltschek, M. Geselle, H. Fuess, Crystal structure and magnetic properties of $\mathrm{CuMoO}_{4}$ at low temperature ( $\gamma$-phase), J. Phys. Chem. Solids 58 (1997) 153-160. doi:10.1016/S0022-3697(96)00108-4.

[26] T. Ito, H. Takagi, T. Asano, Drastic and sharp change in color, shape, and magnetism in transition of $\mathrm{CuMoO}_{4}$ single crystals, Chem. Mater. 21 (2009) 3376-3379. doi:10.1021/cm901029v.

[27] S. Wei, X. Kong, H. Wang, Y. Mao, M. Chao, J. Guo, E. Liang, Negative thermal expansion property of $\mathrm{CuMoO}_{4}$, Optik 160 (2018) 61-67. doi:10.1016/j.ijleo.2018.01.105.

[28] I. Jonane, A. Cintins, A. Kalinko, R. Chernikov, A. Kuzmin, X-ray absorption spectroscopy of thermochromic phase transition in $\mathrm{CuMoO}_{4}$, Low Temp. Phys. 44 (2018) 434-437. doi:10.1063/1.5034155. 
[29] I. Jonane, A. Cintins, A. Kalinko, R. Chernikov, A. Kuzmin, Probing the thermochromic phase transition in $\mathrm{CuMoO}_{4}$ by EXAFS spectroscopy, Phys. Status Solidi B (2018) 1800074doi:10.1002/pssb.20180007.

[30] R. Kohlmuller, J.-P. Faurie, $\mathrm{MoO}_{3}-\mathrm{CuO}$ system, C. R. Acad. Sci., Ser. C 264 (1967) 1751-1752.

[31] J. Timoshenko, A. Kuzmin, J. Purans, EXAFS study of hydrogen intercalation into $\mathrm{ReO}_{3}$ using the evolutionary algorithm, J. Phys.: Condens. Matter 26 (2014) 055401. doi:10.1088/0953-8984/26/5/055401.

[32] J. J. Rehr, R. C. Albers, Theoretical approaches to x-ray absorption fine structure, Rev. Mod. Phys. 72 (2000) 621. doi:10.1103/RevModPhys.72.621.

[33] A. Di Cicco, G. Aquilanti, M. Minicucci, E. Principi, N. Novello, A. Cognigni, L. Olivi, Novel XAFS capabilities at ELETTRA synchrotron light source, J. Phys.: Conf. Ser. 190 (2009) 012043. doi:10.1088/1742-6596/190/1/012043.

[34] B. Ravel, M. Newville, ATHENA, ARTEMIS, HEPHAESTUS: data analysis for X-ray absorption spectroscopy using IFEFFIT, J. Synchrotron Rad. 12 (2005) 537-541. doi:10.1107/S0909049505012719.

[35] J. Timoshenko, A. Kuzmin, Wavelet data analysis of EXAFS spectra, Comp. Phys. Commun. $180 \quad$ (2009) 920-925. doi:10.1016/j.cpc.2008.12.020.

[36] A. L. Ankudinov, B. Ravel, J. J. Rehr, S. D. Conradson, Realspace multiple-scattering calculation and interpretation of X-rayabsorption near-edge structure, Phys. Rev. B 58 (1998) 7565-7576. doi:10.1103/PhysRevB.58.7565.

[37] F. R. Hampel, Robust estimation: a condensed partial survey, Probab. Theory Relat. Field 27 (1973) 87-104. doi:10.1007/BF00536619.

[38] M. Daszykowski, K. Kaczmarek, Y. Vander Heyden, B. Walczak, Robust statistics in data analysis - a review: basic concepts, Chemometrics Intell. Lab. Syst. 85 (2007) 203-219. doi:10.1016/j.chemolab.2006.06.016. 
[39] E. Sevillano, H. Meuth, J. Rehr, Extended x-ray absorption fine structure Debye-Waller factors. I. Monatomic crystals, Phys. Rev. B 20 (1979) 4908. doi:10.1103/PhysRevB.20.4908.

[40] G. Dalba, P. Fornasini, EXAFS Debye-Waller factor and thermal vibrations of crystals, J. Synchrotron Rad. 4 (1997) 243-255. doi:10.1107/S0909049597006900.

[41] Y. Joly, X-ray absorption near-edge structure calculations beyond the muffin-tin approximation, Phys. Rev. B 63 (2001) 125120. doi:10.1103/PhysRevB.63.125120.

[42] O. Bunau, Y. Joly, Self-consistent aspects of X-ray absorption calculations, J. Phys.: Condens. Matter 21 (2009) 345501. doi:10.1088/0953-8984/21/34/345501.

[43] A. Kuzmin, J. Purans, X-ray absorption spectroscopy study of the local environment around tungsten and molybdenum ions in tungstenphosphate and molybdenum-phosphate glasses, Proc. SPIE 2968 (1997) 180-185. doi:10.1117/12.266831.

[44] F. Rocca, A. Kuzmin, P. Mustarelli, C. Tomasi, A. Magistris, XANES and EXAFS at Mo K-edge in $(\mathrm{AgI})_{1-x}\left(\mathrm{Ag}_{2} \mathrm{MoO}_{4}\right)_{x}$ glasses and crystals, Solid State Ionics 121 (1999) 189-192. doi:10.1016/S0167-2738(98)00546-3.

[45] K.-i. Shimizu, H. Maeshima, H. Yoshida, A. Satsuma, T. Hattori, Ligand field effect on the chemical shift in XANES spectra of $\mathrm{Cu}(\mathrm{II})$ compounds, Phys. Chem. Chem. Phys. 3 (2001) 862-866. doi:10.1039/B007276L.

[46] A. Kuzmin, A. Anspoks, A. Kalinko, A. Rumjancevs, J. Timoshenko, L. Nataf, F. Baudelet, T. Irifune, Effect of pressure and temperature on the local structure and lattice dynamics of copper(ii) oxide, Phys. Procedia 85 (2016) 27-35. doi:10.1016/j.phpro.2016.11.077.

[47] O. Durmeyer, J. Kappler, E. Beaurepaire, J. Heintz, M. Drillon, Ti K XANES in superconducting $\mathrm{LiTi}_{2} \mathrm{O}_{4}$ and related compounds, J. Phys.: Condens. Matter 2 (1990) 6127. doi:10.1088/0953-8984/2/28/004. 
[48] S. Nozawa, T. Iwazumi, H. Osawa, Direct observation of the quantum fluctuation controlled by ultraviolet irradiation in $\mathrm{srtio}_{3}$, Phys. Rev. B 72 (2005) 121101. doi:10.1103/PhysRevB.72.121101.

[49] D. Manuel, D. Cabaret, C. Brouder, P. Sainctavit, A. Bordage, N. Trcera, Experimental evidence of thermal fluctuations on the X-ray absorption near-edge structure at the aluminum K edge, Phys. Rev. B 85 (2012) 224108. doi:10.1103/PhysRevB.85.224108.

[50] R. Nemausat, D. Cabaret, C. Gervais, C. Brouder, N. Trcera, A. Bordage, I. Errea, F. Mauri, Phonon effects on x-ray absorption and nuclear magnetic resonance spectroscopies, Phys. Rev. B 92 (2015) 144310. doi:10.1103/PhysRevB.92.144310.

[51] R. Nemausat, C. Gervais, C. Brouder, N. Trcera, A. Bordage, C. CoelhoDiogo, P. Florian, A. Rakhmatullin, I. Errea, L. Paulatto, et al., Temperature dependence of X-ray absorption and nuclear magnetic resonance spectra: probing quantum vibrations of light elements in oxides, Phys. Chem. Chem. Phys. 19 (2017) 6246-6256. doi:10.1039/C6CP08393E.

[52] D. A. Spassky, A. N. Vasil'ev, I. A. Kamenskikh, V. V. Mikhailin, A. E. Savon, Y. A. Hizhnyi, S. G. Nedilko, P. A. Lykov, Electronic structure and luminescence mechanisms in $\mathrm{ZnMoO}_{4}$ crystals, J. Phys.: Condens. Matter 23 (2011) 365501. doi:10.1088/0953-8984/23/36/365501.

[53] D. Spassky, S. Ivanov, I. Kitaeva, V. Kolobanov, V. Mikhailin, L. Ivleva, I. Voronina, Optical and luminescent properties of a series of molybdate single crystals of scheelite crystal structure, Phys. Status Solidi C 2 (2005) 65-68. doi:10.1002/pssc.200460112.

[54] Y. Zhang, N. A. W. Holzwarth, R. T. Williams, Electronic band structures of the scheelite materials $\mathrm{CaMoO}_{4}, \mathrm{CaWO}_{4}$, $\mathrm{PbMoO}_{4}$, and $\mathrm{PbWO}_{4}$, Phys. Rev. B 57 (1998) 12738-12750. doi:10.1103/PhysRevB.57.12738.

[55] L. Cavalcante, E. Moraes, M. Almeida, C. Dalmaschio, N. Batista, J. Varela, E. Longo, M. S. Li, J. Andres, A. Beltran, A combined theoretical and experimental study of electronic structure and optical properties of $\beta-\mathrm{ZnMoO}_{4}$ microcrystals, Polyhedron 54 (2013) 13-25. doi:10.1016/j.poly.2013.02.006. 
(a)

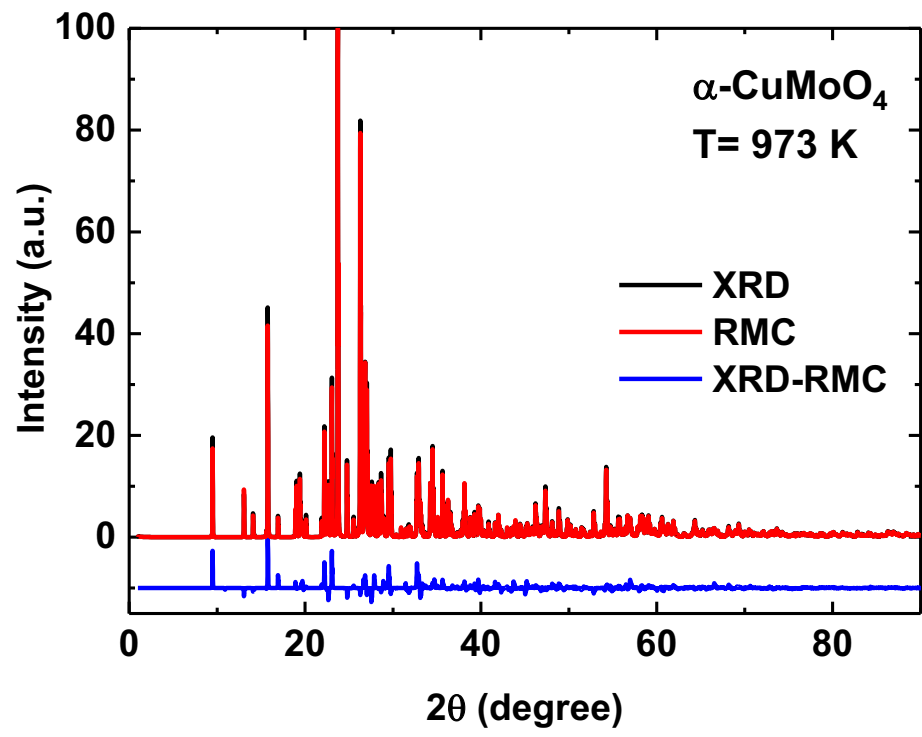

(b)

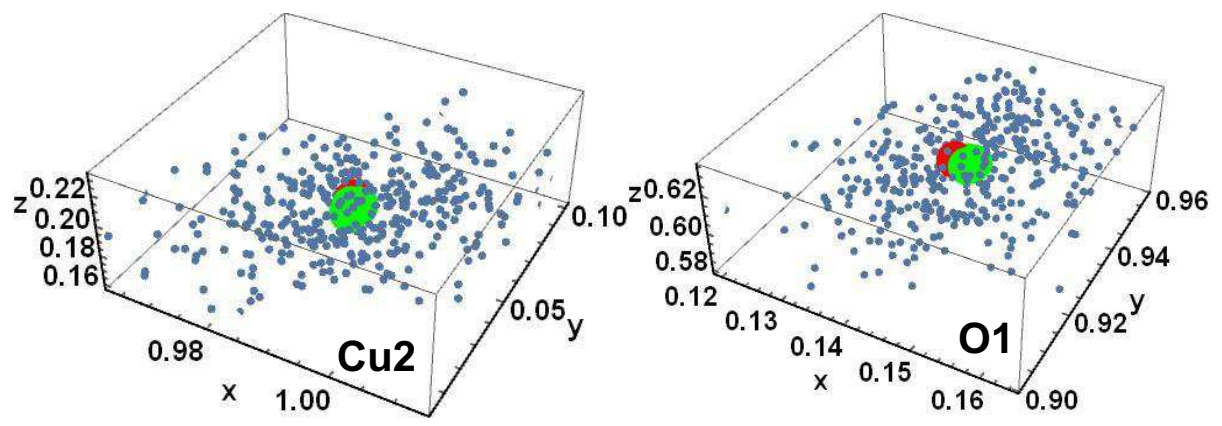

From RMC

Average from RMC

Average from XRD

Figure 9: (a) Example of comparison of diffraction patterns for $\alpha-\mathrm{CuMoO}_{4}$ at $973 \mathrm{~K}$ : black curve - experimental data from [18], red curve - calculation from the average positions of the atoms in the unit cell obtained from the RMC/EA-EXAFS simulation, blue curve - the difference between two patterns. (b) Comparison of the atomic positions (small blue circles) for two representative atoms ( $\mathrm{Cu} 2$ and $\mathrm{O} 1$ ) in the unit cell obtained in the RMC/EA-EXAFS simulations at $973 \mathrm{~K}$ with their Wyckoff positions (red circles) from the diffraction data [18] and evaluated from the RMC/EA simulations (green circles). 

Acta Mater. 179 (2019) 26-35.
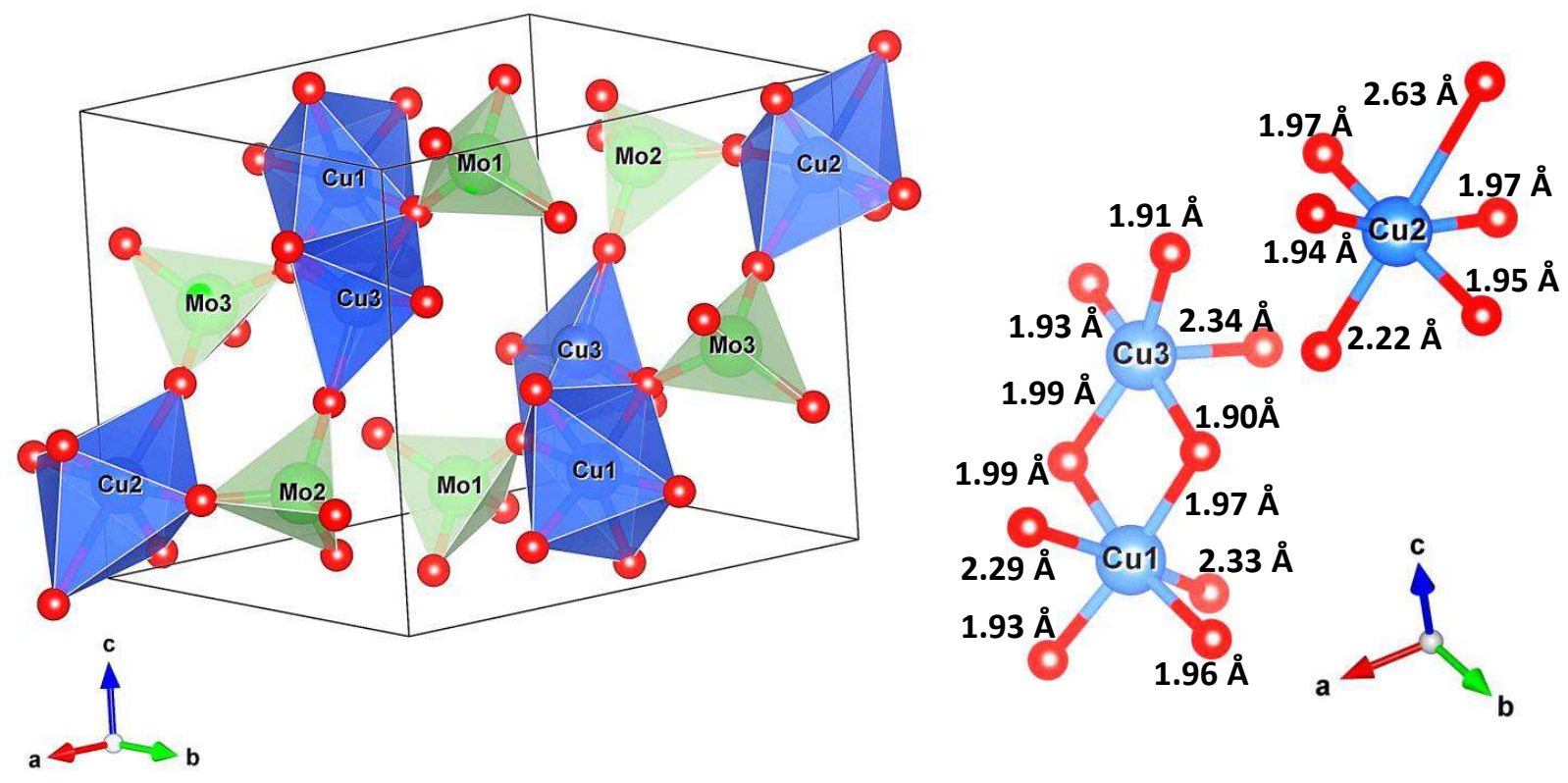

Acta Mater. 179 (2019) 26-35.

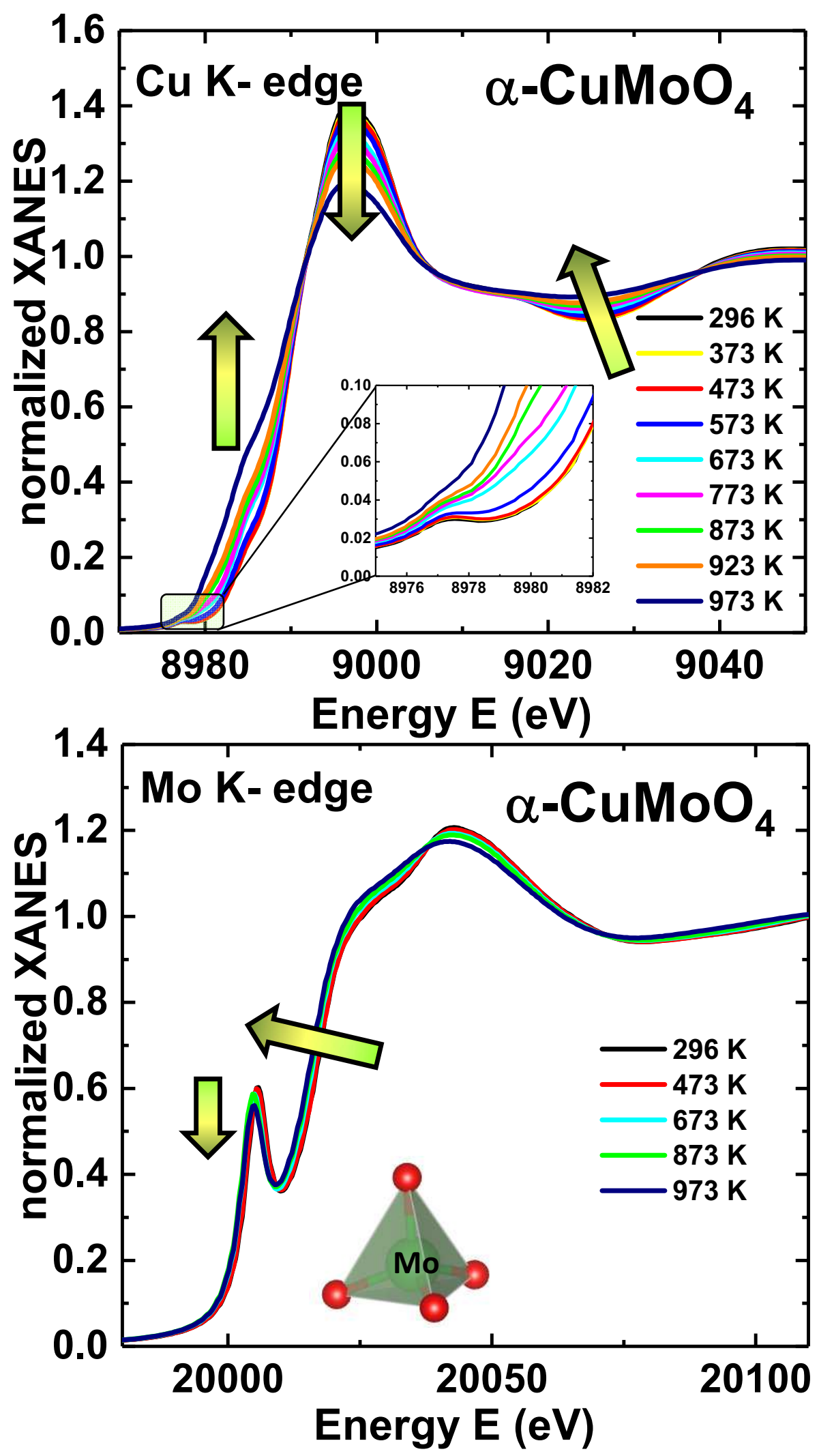



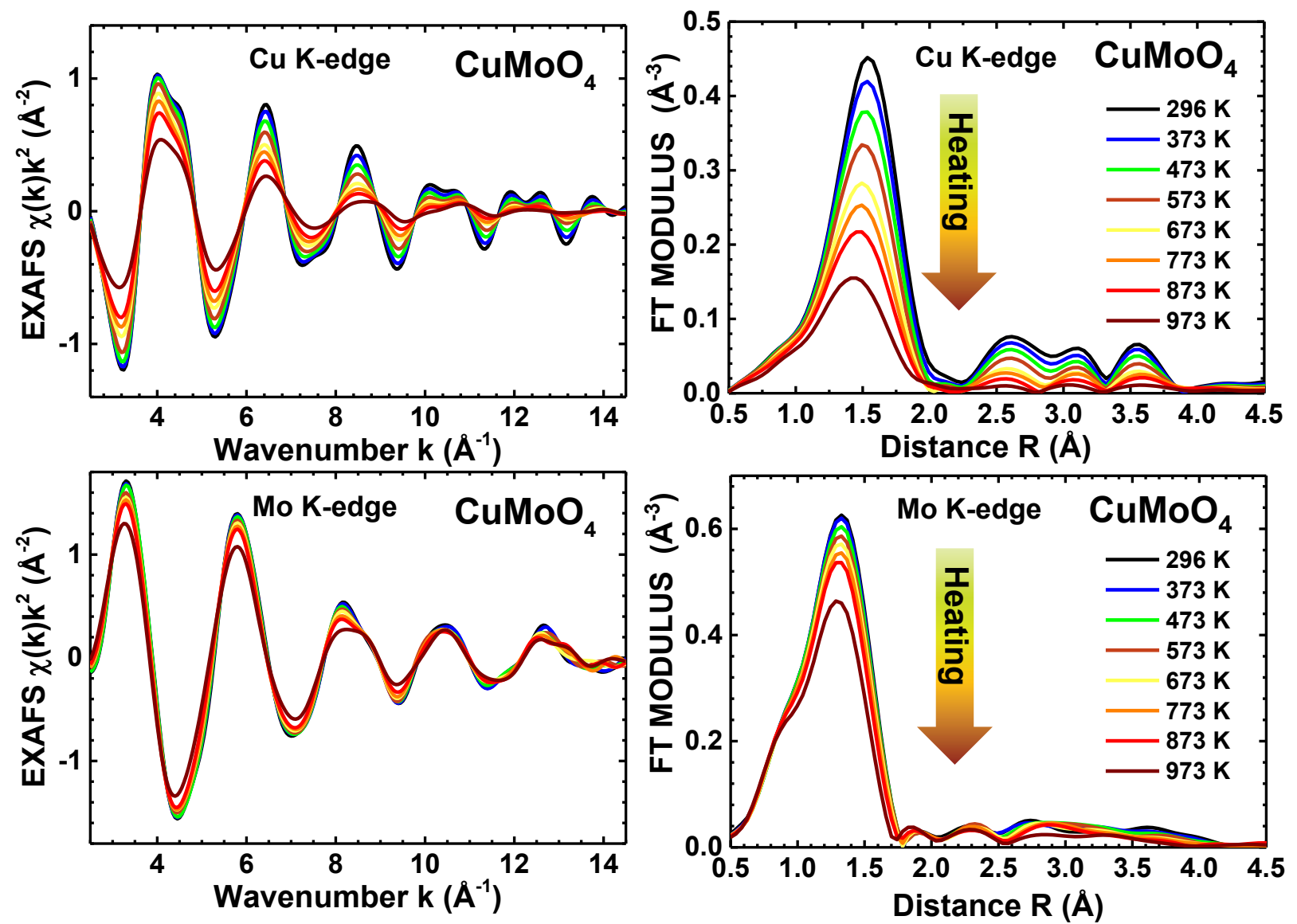

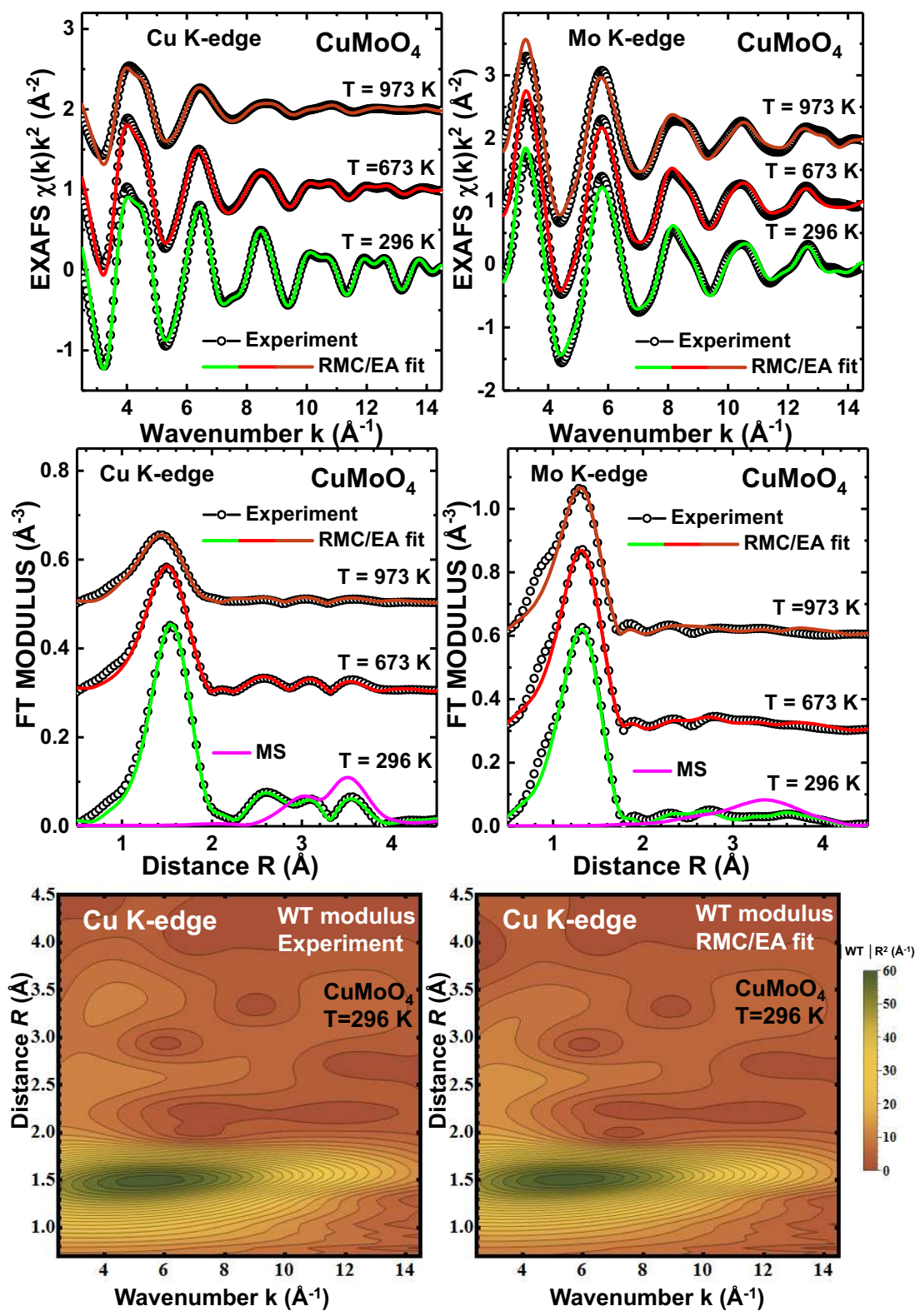
I. Jonane, A. Anspoks, G. Aquilanti, A. Kuzmin,

High-temperature X-ray absorption spectroscopy study of thermochromic copper molybdate, Acta Mater. 179 (2019) 26-35.

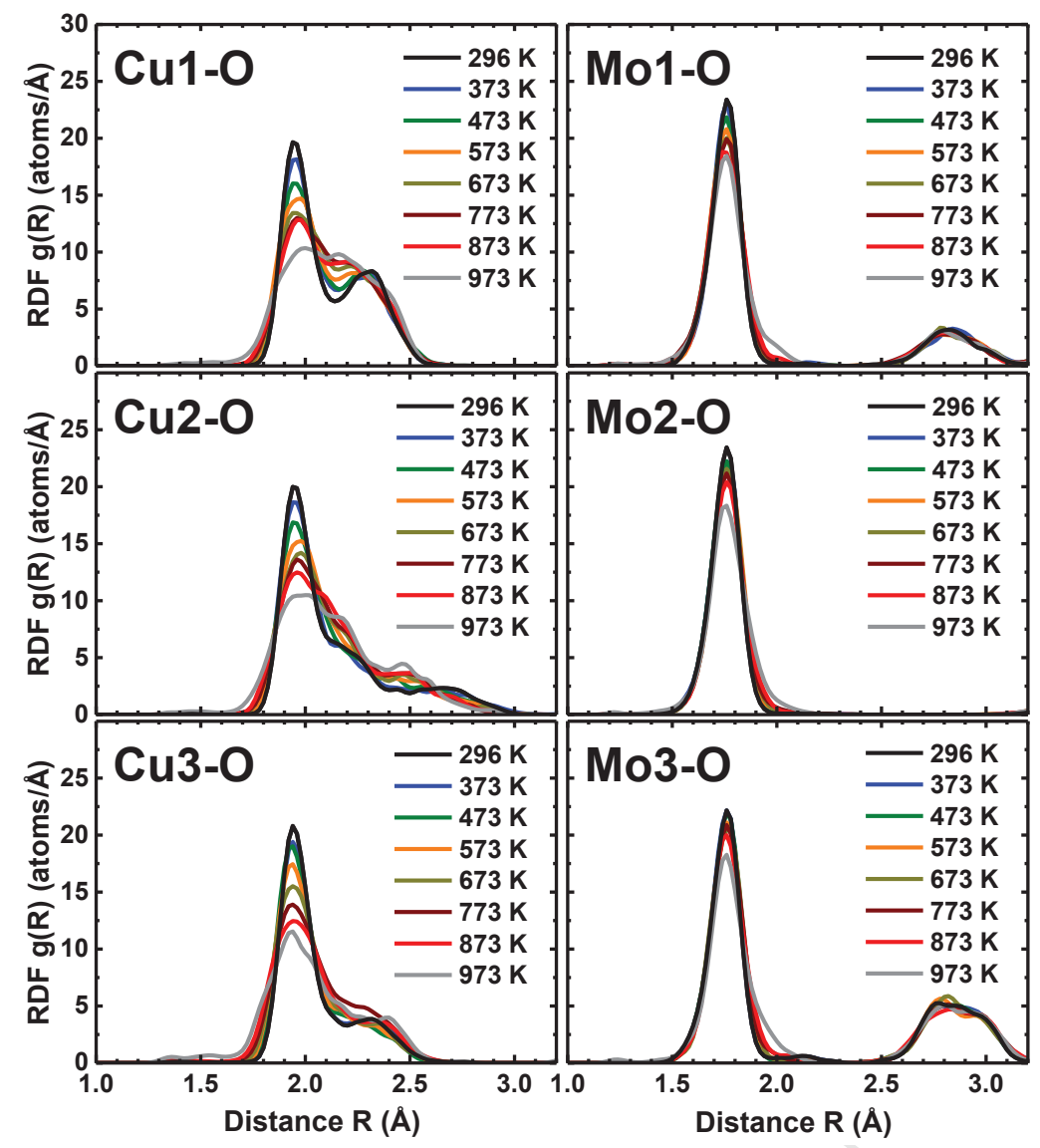



Acta Mater. 179 (2019) 26-35.

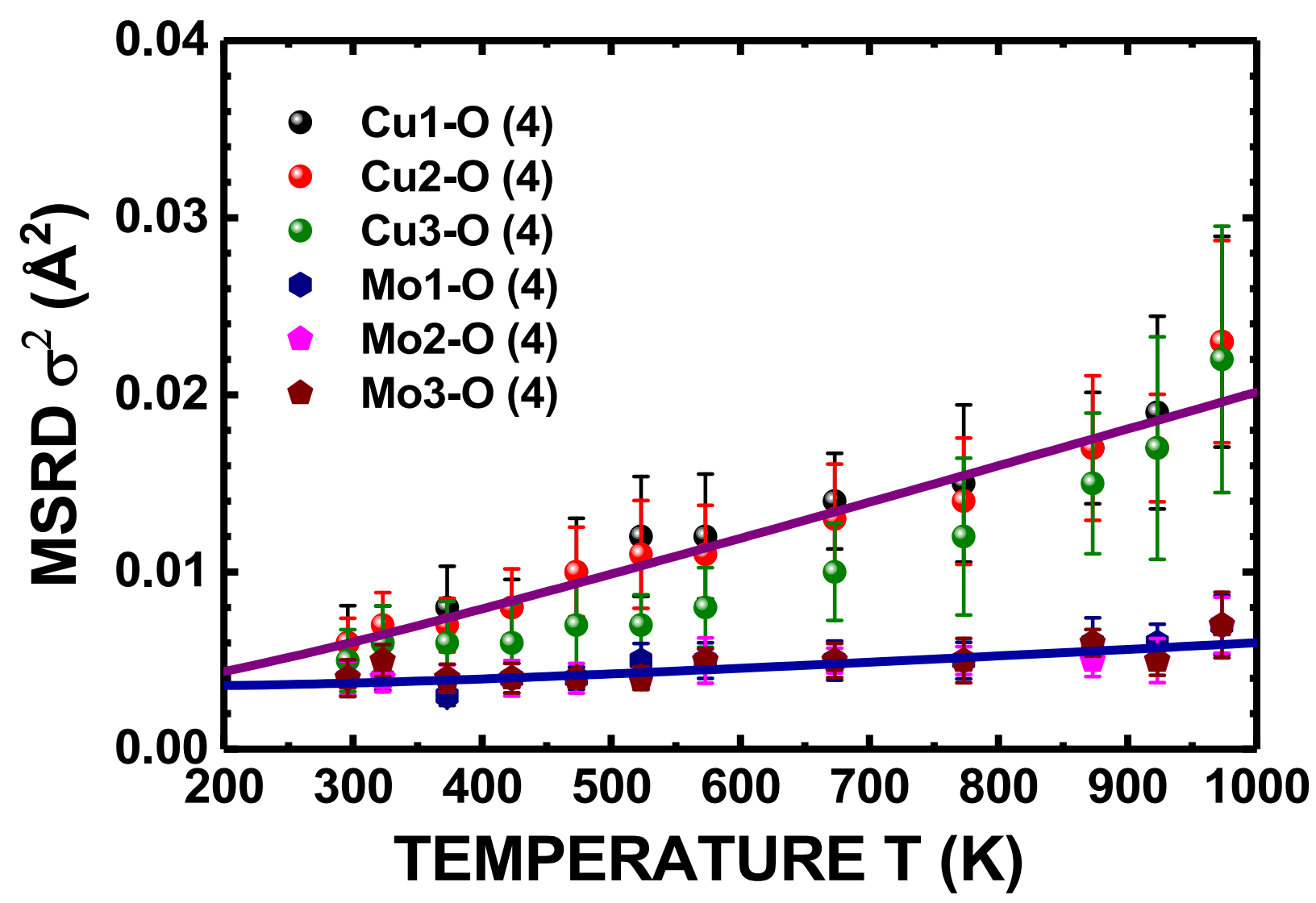



Acta Mater. 179 (2019) 26-35.

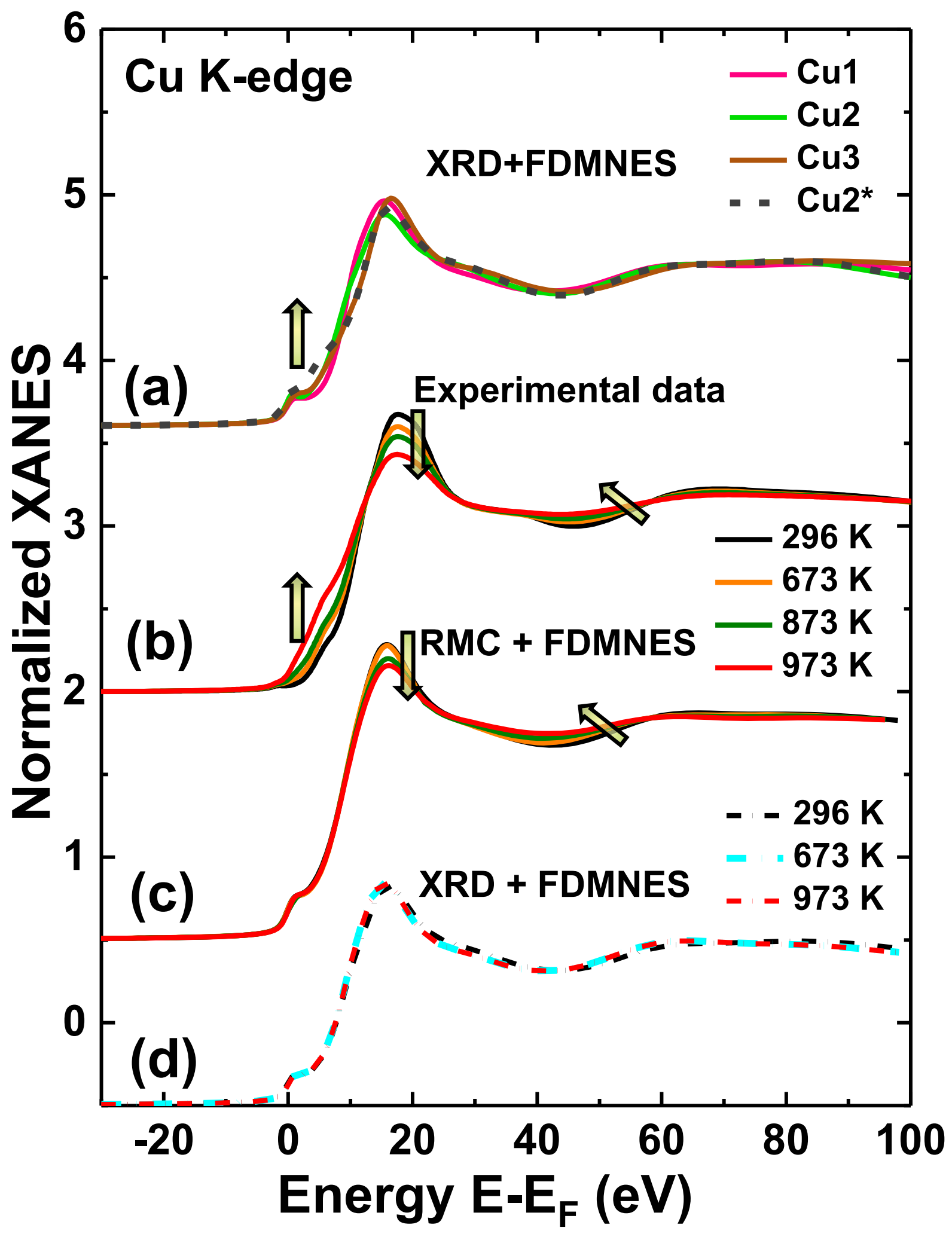



Acta Mater. 179 (2019) 26-35.
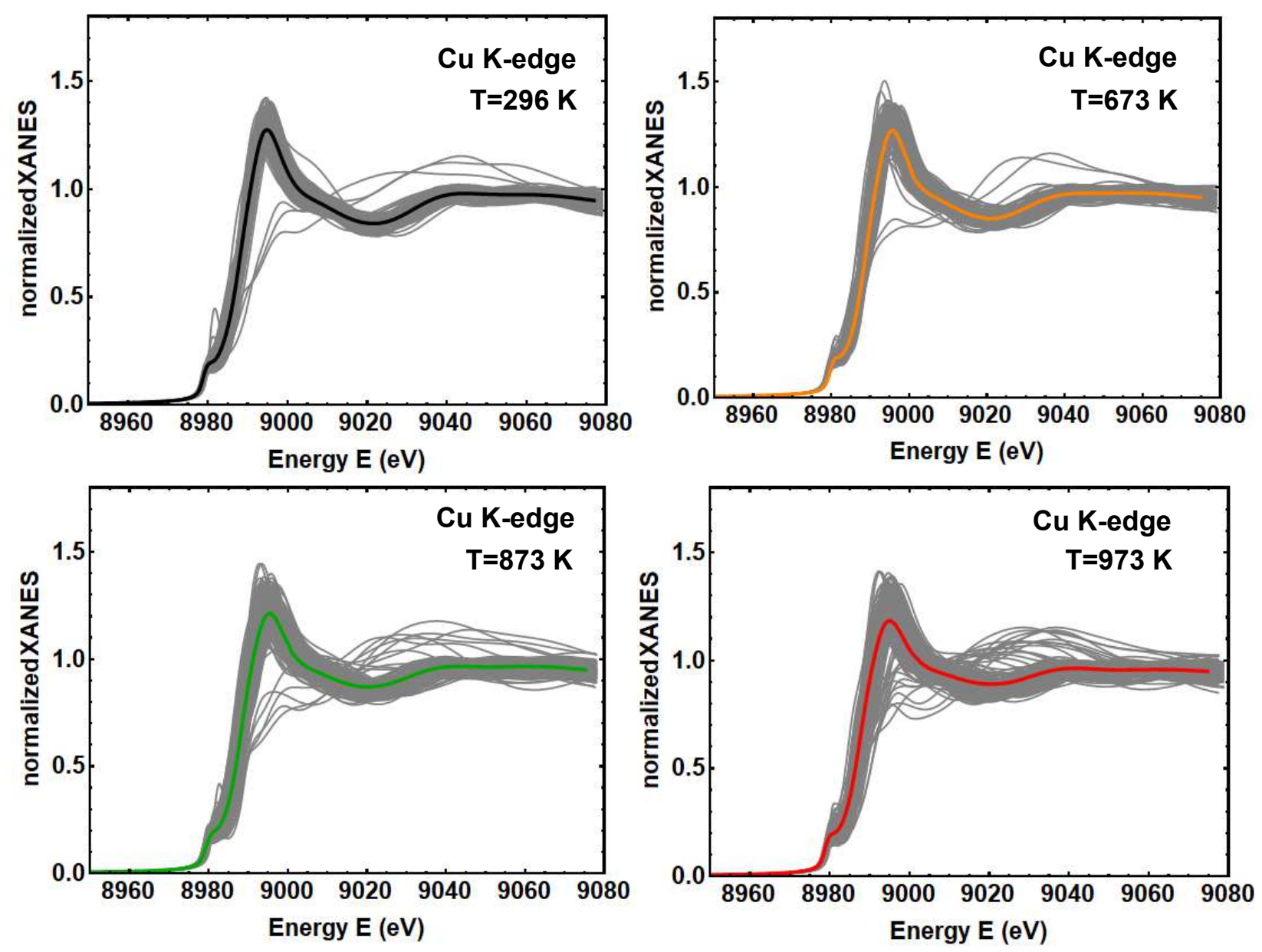

Acta Mater. 179 (2019) 26-35.

(a)

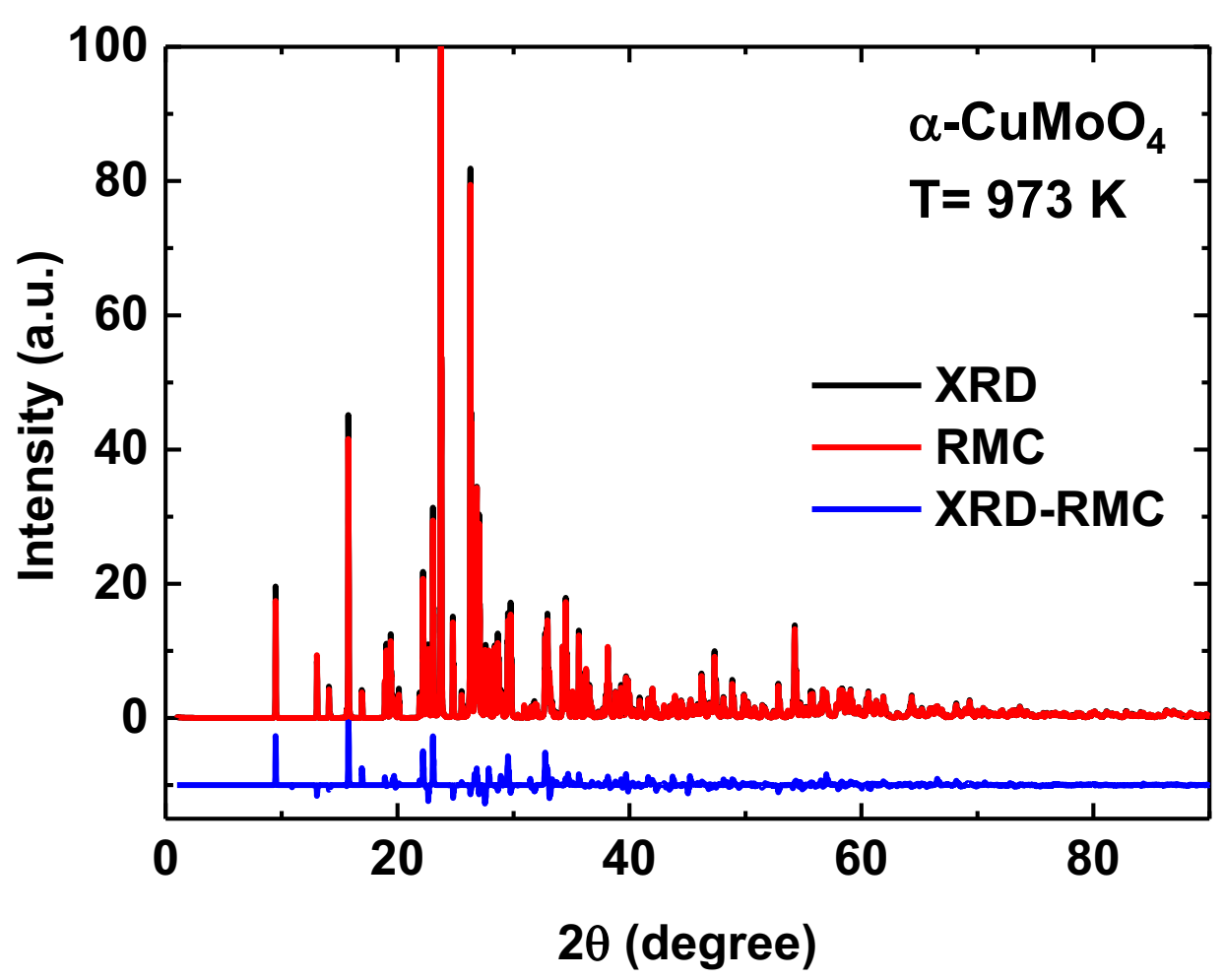

(b)
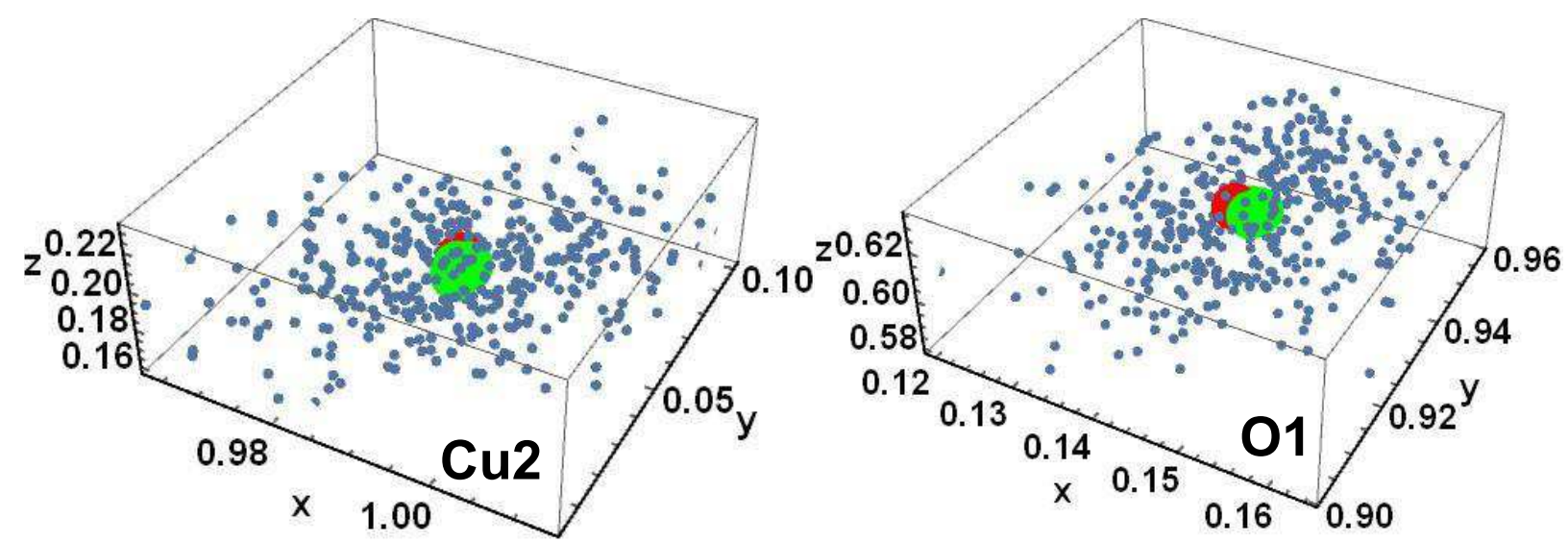

From RMC

Average from RMC

Average from XRD

Institute of Solid State Physics, University of Latvia as the Center of Excellence has received funding from the European Union's Horizon 2020 Framework Programme H2020-WIDESPREAD-01-2016-2017TeamingPhase2 under grant agreement No. 739508, project CAMART2 